\title{
HfS, Hyperfine Structure Fitting Tool
}

\author{
Robert Estalella ${ }^{1}$ \\ Departament de Física Quàntica i Astrofísica (formerly Astronomia i Meteorologia), Institut de Ciències del Cosmos (ICC), Universitat de Barcelona (IEEC-UB), \\ Martí i Franquès 1, E08028 Barcelona, Spain; robert.estalella@ub.edu \\ Received 2016 June 1; accepted 2016 August 9; published 2017 January 12
}

\begin{abstract}
Hyperfine Structure Fitting $(H f S)$ is a tool to fit the hyperfine structure of spectral lines with multiple velocity components. The $H f S \_n h 3$ procedures included in $H f S$ simultaneously fit the hyperfine structure of the $\mathrm{NH}_{3}(J$, $K)=(1,1)$ and $(2,2)$ transitions, and perform a standard analysis to derive $T_{\mathrm{ex}}, \mathrm{NH}_{3}$ column density, $T_{\mathrm{rot}}$, and $T_{\mathrm{k}}$. $H f S$ uses a Monte Carlo approach for fitting the line parameters. Special attention is paid to the derivation of the parameter uncertainties. HfS includes procedures that make use of parallel computing for fitting spectra from a data cube.
\end{abstract}

Key words: ISM: molecules - methods: data analysis

Online material: color figures

\section{Introduction}

Hyperfine Structure (HfS) (Estalella 2016) is a tool to fit the hyperfine structure of spectral lines, with the possibility of fitting simultaneously multiple velocity components. The $H f S \_n h 3$ procedures included in $H f S$ fit simultaneously the hyperfine quadrupole and magnetic structure of the $\mathrm{NH}_{3}$ ( $J$, $K)=(1,1)$ and $(2,2)$ inversion transitions. The assumptions made by $H f S$ are that the beam filling factor $f$, the excitation temperature $T_{\mathrm{ex}}$, the hyperfine lines linewidth $\Delta V$, and the central velocity $V_{\mathrm{LSR}}$ are the same for all of the hyperfine lines.

For $H f S \_n h 3$, these assumptions hold for the hyperfine lines of both $\mathrm{NH}_{3}$ inversion transitions, $(1,1)$ and $(2,2)$. In addition, the results of the fit are used by $H f S_{-} n h 3$ to derive physical parameters, including the excitation temperature; $\mathrm{NH}_{3}$ column density; and the rotational and kinetic temperatures, with the assumption that the emitting region is homogeneous along the line of sight.

$H f S$ is written in Fortran 77 and 90/95 (mainly for the dynamical storage of arrays). The graphic interface uses the PGplot Graphics Subroutine Library ${ }^{2}$, and some procedures have a multiprocessor version running under Open MPI. ${ }^{3}$ The interactive procedures are menu driven, allowing to select options with the keyboard and, for some actions, with the mouse cursor and buttons.

One of the advantages of $H f S$ when compared with other packages performing hyperfine fitting (e.g., the widely used CLASS of GILDAS ${ }^{4}$ ), is that it is well documented, easy to

\footnotetext{
1 The ICC (UB) is a CSIC-Associated Unit through the ICE (CSIC).

2 http://www.astro.caltech.edu/ tjp/pgplot/

3 http://www.open-mpi.org

4 https://www.iram.fr/IRAMFR/GILDAS/
}

install, and has a simple interface. In a single run, $H f S$ can fit multiple velocity components to the spectra of a FITS data cube and obtain the maps of the parameters fitted and derived from the fit in a short computing time, taking advantage of the multiple processors of current computers.

A preliminary version of $H f S$ was briefly described and used for fitting $\mathrm{NH}_{3}(1,1)$ and $(2,2)$ spectra in Sánchez-Monge et al. (2013).

The structure of the paper is as follows. The fit parameters used by $H f S$ are described in Section 2, the fitting strategy in Section 3, the calculation of the synthetic spectrum in Section 4, the line parameters derived from the fit parameters in Section 5, the $\mathrm{NH}_{3}$ physical parameters derived by $H f S \_n h 3$ in Section 6, the error estimation for the fit parameters in Section 7, and the derived parameters in Section 8. A comparison with the results obtained with CLASS and the Rosolowsky et al. (2008) routine is presented in Section 9. The different procedures that compose $H f S$ are described in Section 10. Finally, in several Appendices we describe the requisites and installation instructions (Appendices A to C) and examples of the use of $H f S$ and of input and output files (Appendices D to G).

\section{Fit Parameters}

For every velocity component of a transition with hyperfine structure, the general $H f S$ procedures fit simultaneously four independent parameters:

- $\Delta V$, hyperfine lines linewidth, assumed to be the same for all the hyperfine lines,

- $V_{\text {LSR }}$, main line central LSR velocity,

- $A_{m}^{*} \equiv A\left(1-\exp \left\{-\tau_{m}\right\}\right)$, main line peak intensity (for hyperfine lines wider than the hyperfine separation and the 
Table 1

Relative Velocity $V_{\text {hyp }}$, and Optical Depth $\tau_{\text {hyp }}$ with Respect to that of the Main Line, $\tau_{m}$, of the Hyperfine Lines of the $\mathrm{NH}_{3}(J, K)=(1,1)$ and $(2,2)$ Inversion Transitions (Mangum \& Shirley 2015)

\begin{tabular}{|c|c|c|c|c|}
\hline \multirow{3}{*}{$\frac{\mathrm{os}^{\mathrm{a}}}{}$} & \multicolumn{2}{|c|}{$(J, K)=(1,1)$} & \multicolumn{2}{|c|}{$(J, K)=(2,2)$} \\
\hline & $\begin{array}{c}V_{\text {hyp }} \\
\left(\mathrm{km} \mathrm{s}^{-1}\right)\end{array}$ & $\tau_{\text {hyp }}$ & $\begin{array}{c}V_{\text {hyp }} \\
\left(\mathrm{km} \mathrm{s}^{-1}\right)\end{array}$ & $\tau_{\text {hyp }}$ \\
\hline & $\begin{array}{l}-19.548593 \\
-19.409429\end{array}$ & $\begin{array}{l}0.148148 \\
0.074074\end{array}$ & $\begin{array}{l}-26.557749 \\
-26.042011 \\
-25.981267\end{array}$ & $\begin{array}{l}0.004186 \\
0.037674 \\
0.020930\end{array}$ \\
\hline is $^{b}$ & $\begin{array}{l}-7.815393 \\
-7.373698 \\
-7.234546\end{array}$ & $\begin{array}{l}0.166667 \\
0.018519 \\
0.092593\end{array}$ & $\begin{array}{l}-16.401673 \\
-16.389235 \\
-15.873510\end{array}$ & $\begin{array}{l}0.037209 \\
0.026047 \\
0.001860\end{array}$ \\
\hline $\mathrm{m}^{\mathrm{c}}$ & $\begin{array}{r}-0.252188 \\
-0.213155 \\
-0.133040 \\
-0.073991 \\
0.189495 \\
0.308643 \\
0.323117 \\
0.462268\end{array}$ & $\begin{array}{l}0.033333 \\
0.092593 \\
0.466667 \\
0.018519 \\
0.300000 \\
0.033333 \\
0.018519 \\
0.037037\end{array}$ & $\begin{array}{r}-0.589779 \\
-0.531971 \\
-0.502427 \\
-0.013336 \\
-0.003910 \\
0.013298 \\
0.524366 \\
0.529035 \\
0.563171\end{array}$ & $\begin{array}{l}0.020930 \\
0.010631 \\
0.011628 \\
0.146512 \\
0.499668 \\
0.267442 \\
0.011628 \\
0.010631 \\
0.020930\end{array}$ \\
\hline is $^{b}$ & $\begin{array}{l}7.350050 \\
7.469198 \\
7.886323\end{array}$ & $\begin{array}{l}0.018519 \\
0.166667 \\
0.092593\end{array}$ & $\begin{array}{l}15.883012 \\
16.398737 \\
16.411175\end{array}$ & $\begin{array}{l}0.001860 \\
0.026047 \\
0.037209\end{array}$ \\
\hline $\mathrm{os}^{\mathrm{a}}$ & $\begin{array}{l}19.319597 \\
19.845140\end{array}$ & $\begin{array}{l}0.148148 \\
0.074074\end{array}$ & $\begin{array}{l}25.981267 \\
26.042011 \\
26.557749\end{array}$ & $\begin{array}{l}0.020930 \\
0.037674 \\
0.004186\end{array}$ \\
\hline Sum & & 2.000000 & & 1.255814 \\
\hline
\end{tabular}

Notes.

as: outer satellite.

b is: inner satellite.

c m: main

channel width), where $A$ is the amplitude (see Section 5), and

- $\tau_{m}^{*} \equiv 1-\exp \left\{-\tau_{m}\right\}$, where $\tau_{m}$ is the optical depth of the main line.

The $H f S \_n h 3$ procedures included in $H f S$ fit simultaneously the hyperfine structure of a pair of spectra of the $\mathrm{NH}_{3}$ inversion transitions $(J, K)=(1,1)$ and $(2,2)$ (see Table 1$)$.

Differences in central velocity of the order of a tenth of $\mathrm{km} \mathrm{s}^{-1}$ are usually found between the $(1,1)$ and $(2,2)$ emissions (e.g., Sepúlveda et al. 2011). Thus, a different $V_{\text {LSR }}$ is fitted for the $(1,1)$ and the $(2,2)$ spectra, resulting in two additional parameters for the $(2,2)$ transition, fitted simultaneously with the four parameters for the $(1,1)$ transition, $\Delta V$, $V_{\mathrm{LSR} 1}, A_{1 m}^{*}, \tau_{1 m}^{*}$, already described:

- $V_{\mathrm{LSR} 2}$, central LSR velocity of the $(2,2)$ transition, and
- $A_{2 m}^{*} \equiv A\left(1-\exp \left\{-\tau_{2 m}\right\}\right)$, peak intensity of the $(2,2)$ main line (for hyperfine lines wider than the hyperfine separation and the channel width).

For each set of fit parameters, the optical depth of the $(2,2)$ main line is obtained from the relation

$$
\tau_{2 m}^{*} \equiv 1-\exp \left\{-\tau_{2 m}\right\}=\tau_{1 m}^{*} \frac{A_{2 m}^{*}}{A_{1 m}^{*}} .
$$

The use of $A_{m}^{*} \equiv A\left(1-\exp \left\{-\tau_{m}\right\}\right) \quad$ and $\quad \tau_{m}^{*} \equiv 1-$ $\exp \left\{-\tau_{m}\right\}$ as fit parameters instead of $A$ or $A \tau_{m}$, and $\tau_{m}$ needs some justification. First, $A_{m}^{*}$ is well determined even in the two limiting cases, $\tau_{m} \ll 1$ (with $A$ ill determined) and $\tau_{m} \gg 1$ (with $A \tau_{m}$ ill determined). This is because $A_{m}^{*}$ is a true physical magnitude, i.e., the peak intensity of the main line, provided that the the hyperfine lines that may compose the main line are blended. Second, $\tau_{m}^{*}$ is bounded, $0<\tau_{m}^{*}<1$, and the two bounds correspond to the optically thin and thick limiting cases, where the fit is degenerate and no longer depends on $\tau_{m}$. In short, the two fit parameters are well determined in all cases, even in the optically thin and thick limits. The only drawback of the use of $\tau_{m}^{*}$ as fit parameter is for the case of extremely high opacity in the simultaneous fit of $\mathrm{NH}_{3}(1,1)$ and $(2,2)$, when the intensity of the $(2,2)$ main and satellite lines is similar. In its present version, $H f S \_n h 3$ is not able to accurately deal with $\tau_{1 m}>16$, but this case is indeed very rare. In a survey of low-mass cores in Perseus (Rosolowsky et al. 2008), none of the sources has such a high value of optical depth, and in another survey of high-mass clumps in the Galactic Plane (Svoboda et al. 2016), only $1 \%$ of the sources show $\tau_{1 m}>16$. Thus, HfS_nh3 should work for the vast majority of cases.

The fitting procedure ends with a set of four or, for the $H f S \_n h 3$ procedures, six values plus $\tau_{2 m}^{*}$, of the fit parameters for every velocity component, which minimize the fit residual $\chi^{2}$ of the spectrum (see Section 3 ) and an estimation of the uncertainty of the fit parameters (see Section 7), $\sigma(\Delta V), \sigma$ $\left(V_{\mathrm{LSR}}\right), \sigma\left(A_{m}^{*}\right), \sigma\left(\tau_{m}^{*}\right)$, and, for the $H f S \_n h 3$ procedures, $\sigma$ $\left(V_{\mathrm{LSR} 2}\right)$, and $\sigma\left(A_{2 m}^{*}\right)$.

\section{Fitting Strategy}

The fitting procedure is similar to that used in other fitting problems by Estalella et al. (2012) and Palau et al. (2014). HfS samples the space parameter of dimension $m$ (four or six times the number of velocity components), defined by the parameters $p_{1}, \ldots, p_{m}$, to find the minimum value of the fit residual $\chi^{2}$ :

$$
\chi^{2}=\sum_{i=1}^{N}\left[\frac{y_{i}^{\mathrm{obs}}-y_{i}^{\mathrm{mod}}\left(p_{1}, \ldots, p_{m}\right)}{\sigma_{i}}\right]^{2},
$$

where $y_{i}^{\text {obs }}$ are the observed line intensities for a total of $N$ spectral channels, $y_{i}^{\text {mod }}\left(p_{1}, \ldots, p_{m}\right)$ are the model line intensities depending on $m$ free parameters, and $\sigma_{i}$ are the errors of the observations. 
The sampling strategy is based on that used in the asexual genetic algorithm (AGA) (Cantó et al. 2009). The procedure starts with a number of samples of the parameter space within the initial search range for each parameter (seeds). For each seed, the residual $\chi^{2}$ is computed for a number of samples (descendants) within the search range centered on the seed value. The best samples, i.e., those with the lowest $\chi^{2}$, are kept as seeds for the next loop, for which the search ranges are decreased by a constant factor. The procedure is iterated and is stopped after a given number of loops.

Several sampling methods of the $m$-dimensional parameter space are possible, i.e., regular grid, random, Halton or Sobol pseudo-random sequences (Halton 1964; Sobol 1967). HfS uses a Sobol pseudo-random sequence because it samples the $m$-dimensional parameter space more evenly than a purely random sequence, even for high values of $m$, and the convergence of the fitting procedure to the minimum of $\chi^{2}$ is faster.

\section{Synthetic Spectrum}

Let us assume that the transition being fitted has $n_{h}$ hyperfine lines, and for each hyperfine $j=1, \ldots n_{h}, V_{\text {hyp }}^{j}$ is the velocity shift with respect to the main line, and $\tau_{\text {hyp }}^{j}$ is the ratio of the optical depth of the hyperfine and that of the main line, $\tau_{m}$. Let $n_{c}$ be the number of velocity components fitted, and for each velocity component $i=1, \ldots n_{c}, \Delta V^{i}, V_{\mathrm{LSR}}{ }^{i}, A^{i}$, and $\tau_{m}^{i}$ are the values of the fit or derived line parameters (see Section 5).

For each velocity channel $k=1, \ldots N$, with central velocity $V_{k}$ and channel width $\Delta V_{\mathrm{ch}}$, the contribution of the velocity component $i$ to the optical depth is calculated as

$$
\tau_{k}^{i}=\sum_{j=1}^{n_{h}} \frac{\tau_{m}^{i} \tau_{\text {hyp }}^{j}}{\Delta V_{\mathrm{ch}}} G,
$$

where $G$ is the integral over the velocity range of the channel,

$$
G=\int_{V_{k}-\Delta V_{\text {ch }} / 2}^{V_{k}+\Delta V_{\mathrm{ch}} / 2} e^{-4 \ln 2\left[\left(v-V_{\mathrm{LSR}}^{i}-V_{\text {hyp }}^{j}\right) / \Delta V^{i}\right]^{2}} d v .
$$

$G$ is evaluated by means of the error function $\operatorname{erf}(x)=2 / \sqrt{\pi} \int_{0}^{x} \exp \left(-t^{2}\right) d t$ as

$$
G=\frac{\sqrt{\pi}}{4 \sqrt{\ln 2}} \Delta V^{i}\left[\operatorname{erf}\left(x^{+}\right)-\operatorname{erf}\left(x^{-}\right)\right],
$$

where

$$
x^{ \pm}=2 \sqrt{\ln 2} \frac{V_{k} \pm \Delta V_{\mathrm{ch}} / 2-V_{\mathrm{LSR}}^{i}-V_{\mathrm{hyp}}^{j}}{\Delta V^{i}} .
$$

If the difference between $x^{+}$and $x^{-}$is too low, within less than 1 part in $10^{4}$, Equation (5) can produce a large roundoff error, and $G$ is evaluated as

$$
G=\frac{\Delta V^{i}}{2 \sqrt{\ln 2}}\left(x^{+}-x^{-}\right) e^{-\left[\left(x^{+}+x^{-}\right) / 2\right]^{2}} .
$$

Finally, the intensity of the channel $k$ of the synthetic spectrum is calculated as the sum of intensities of the different velocity components,

$$
T_{k}=\sum_{i=1}^{n_{c}} A^{i}\left(1-e^{-\tau_{k}^{i}}\right)
$$

\section{Derived Line Parameters}

From the values of the fit parameters, the following derived line parameters are calculated that are necessary for calculating the synthetic spectra and the estimation of parameters with physical interest:

$A$, amplitude. The amplitude is

$$
A=f\left[J_{\nu}\left(T_{\mathrm{ex}}\right)-J_{\nu}\left(T_{\mathrm{bg}}\right)\right],
$$

where $f$ is the beam filling factor, $T_{\mathrm{ex}}$ the excitation temperature, $T_{\text {bg }}$ the background temperature, and

$$
J_{\nu}(T)=\frac{h \nu / k}{e^{h \nu / k T}-1},
$$

is the Planck-corrected temperature. $A$ is calculated from

$$
A=\frac{A_{m}^{*}}{\tau_{m}^{*}} .
$$

In the case of $H f S \_n h 3$, the excitation temperature $T_{\mathrm{ex}}$ and the filling factor $f$ are assumed to be the same for the $(1,1)$ and $(2$, 2 ) lines. Since the frequencies of both transitions are very close (see Table 2), these assumptions imply that the amplitude $A$ is the same for both lines, within less than 1 in $10^{4}$.

$\tau_{m}$, optical depth of the main line. Calculated from

$$
\tau_{m}=-\ln \left(1-\tau_{m}^{*}\right) .
$$

Special care has to be taken when $\tau_{m} \simeq \tau_{m}^{*} \ll 1$, since the last expression involves the difference of 1 and a small number. In this case, a good approximation is the Taylor expansion,

$$
\tau_{m} \simeq \tau_{m}^{*}+\frac{1}{2} \tau_{m}^{* 2}+\frac{1}{3} \tau_{m}^{* 3} .
$$

In the case of $H f S \_n h 3, \tau_{1 m}$ and $\tau_{2 m}$ are derived in the same way.

$A \tau_{m}$, amplitude times the main line optical depth. Calculated from

$$
A \tau_{m}=A_{m}^{*} \frac{\tau_{m}}{\tau_{m}^{*}} .
$$

Note that for $\tau_{m}^{*} \ll 1, A \tau_{m} \simeq A_{m}^{*}$. In the case of $H f S_{-} n h 3, A \tau_{1 m}$ and $A \tau_{2 m}$ are derived in the same way.

\section{6. $\mathrm{NH}_{3}$ Derived Physical Parameters}

In addition to the derived line parameters, $H f S \_n h 3$ derives physical parameters using the standard analysis of $\mathrm{NH}_{3}(1,1)$ and $(2,2)$ observations, which assumes that the region observed is homogeneous along the line of sight. The physical 
Table 2

Values of the $\mathrm{NH}_{3}(1,1)$ and $(2,2)$ Inversion Transition Frequencies (Kukolich 1967), Spontaneous Emission Einstein Coefficients (Osorio et al. 2009), Ratios of Total to Main Line Optical Depths (Mangum \& Shirley 2015), and $B(J, K)$ and $C(J, K)$ Coefficients of Anglada et al. (1995) Recalculated with the Improved Values of the Constants

\begin{tabular}{lccccc}
\hline \hline & $\nu_{j k}$ & $T_{\nu}=h \nu_{j k} / k$ & $A_{j k}$ & $R_{m}=$ & \\
$(J, K)$ & $(\mathrm{GHz})$ & $(\mathrm{K})$ & $\left(10^{-7} \mathrm{~s}^{-1}\right)$ & $\tau_{\text {tot }} / \tau_{m}$ & $B(J, K)$ \\
\hline$(1,1)$ & 23.69450 & 1.137157 & 1.66838 & 2.000000 & $1.58339 \times 10^{13}$ \\
$(2,2)$ & 23.72263 & 1.138507 & 2.23246 & 1.255814 & $7.45665 \times 10^{12}$ \\
\hline
\end{tabular}

parameters derived are the excitation temperature $T_{\mathrm{ex}}$; the $\mathrm{NH}_{3}(1,1)$ and $(2,2)$ beam-averaged column densities $f N(1,1)$ and $f N(2,2)$; the rotational temperature $T_{\text {rot }}$; the $\mathrm{NH}_{3}$ beamaveraged column density $f N\left(\mathrm{NH}_{3}\right)$; and the kinetic temperature $T_{k}$

Excitation temperature. The excitation temperature is obtained from the amplitude $A$,

$$
T_{\mathrm{ex}}=\frac{T_{\nu}}{\ln \left[1+\frac{T_{\nu}}{A / f+J_{\nu}\left(T_{\mathrm{bg}}\right)}\right]},
$$

where $T_{\nu}=h \nu / k$ is the frequency in temperature units. The frequency of the $(1,1)$ transition is used, but since the frequency of the $(2,2)$ transition is very close (see Table 2 ), the result does not depend on which of the two frequencies is used. For values of $T_{\mathrm{ex}} \gg T_{\mathrm{bg}}>T_{\nu}$, this expression simplifies to

$$
T_{\mathrm{ex}} \simeq A / f \text {. }
$$

The value of the excitation temperature depends on the value assumed for the filling factor $f$. The usual assumption is that the filling factor $f=1$. The value obtained with this assumption is a lower limit for the value of $T_{\mathrm{ex}}$. On the contrary, if we assume that $f \ll 1, T_{\mathrm{ex}} \rightarrow \infty$.

$\mathrm{NH}_{3}(1,1)$ and $(2,2)$ column densities. The column density of the $(J, K)=(1,1)$ and $(2,2)$ levels (i.e., the sum of column densities of the two inversion levels of the corresponding ( $J$, $K$ ) rotational level) can be given as (Anglada et al. 1995; Estalella \& Anglada 1997)

$$
N(J, K)=\sqrt{\frac{\pi}{4 \ln 2}} \frac{8 \pi \nu_{j k}^{3}}{c^{3} A_{j k}} R_{m} \frac{\exp \left(T_{\nu} / T_{\mathrm{ex}}\right)+1}{\exp \left(T_{\nu} / T_{\mathrm{ex}}\right)-1} \tau_{m} \Delta V,
$$

where $A_{j k}$ is the Einstein coefficient of the inversion transition of the rotational level $(J, K) ; R_{m}=\tau_{\text {tot }} / \tau_{m}$ is the ratio of total and main line optical depths of the inversion transition; and $T_{\nu}=h \nu_{J K} / k$ is the frequency of the inversion transition in temperature units (see Table 2).

The last expression depends on the value of the filling factor $f$ assumed to derive $T_{\mathrm{ex}}$. The explicit dependence on $f$ is

$$
\frac{\exp \left(T_{\nu} / T_{\mathrm{ex}}\right)+1}{\exp \left(T_{\nu} / T_{\mathrm{ex}}\right)-1}=\frac{2}{f T_{\nu}}\left(A+f\left[J_{\nu}\left(T_{\mathrm{bg}}\right)+T_{\nu} / 2\right]\right)
$$

so that the beam-averaged column density can be expressed as

$$
\begin{aligned}
f N(J, K)= & \sqrt{\frac{\pi}{4 \ln 2}} \frac{16 \pi k \nu_{j k}^{2}}{h c^{3} A_{j k}} R_{m} \\
& \times\left(A \tau_{m}+f\left[J_{\nu}\left(T_{\mathrm{bg}}\right)+\frac{T_{\nu}}{2}\right] \tau_{m}\right) \Delta V,
\end{aligned}
$$

The maximum value of $f N(J, K)$ is obtained for $f=1$ (the usual assumption to derive $T_{\mathrm{ex}}$ ), while the minimum value is obtained for $f \ll 1$. In the latter case (or for $A \simeq T_{\mathrm{ex}} \gg T_{\mathrm{bg}}>T_{\nu}$ ), the expression simplifies to

$$
f N(J, K)=\sqrt{\frac{\pi}{4 \ln 2}} \frac{16 \pi k \nu_{j k}^{2}}{h c^{3} A_{j k}} R_{m} A \tau_{m} \Delta V .
$$

The values of the constants appearing in these equations are given in Table 2.

In practical units, the two equations become (Anglada et al. 1995)

$$
\left[\frac{N(J, K)}{\mathrm{cm}^{2}}\right]=B(J, K) \frac{\exp \left(T_{\nu} / T_{\mathrm{ex}}\right)+1}{\exp \left(T_{\nu} / T_{\mathrm{ex}}\right)-1} \tau_{m}\left[\frac{\Delta V}{\mathrm{~km} \mathrm{~s}^{-1}}\right]
$$

and, for $f \ll 1$, or $T_{\text {ex }} \gg T_{\text {bg }}>T_{\nu}$,

$$
\left[\frac{f N(J, K)}{\mathrm{cm}^{2}}\right]=C(J, K) A \tau_{m}\left[\frac{\Delta V}{\mathrm{~km} \mathrm{~s}^{-1}}\right],
$$

The values of the constants $B(J, K)$ and $C(J, K)$ for the $(1,1)$ and $(2,2)$ transitions are given in Table 2 . The expression equivalent to these two equations, with an explicit dependence on $f$, is

$$
\begin{aligned}
{\left[\frac{f N(J, K)}{\mathrm{cm}^{2}}\right]=} & C(J, K)\left(A \tau_{m}+f\left[J_{\nu}\left(T_{\mathrm{bg}}\right)+\frac{T_{\nu}}{2}\right] \tau_{m}\right) \\
& \times\left[\frac{\Delta V}{\mathrm{~km} \mathrm{~s}^{-1}}\right] .
\end{aligned}
$$

Rotational temperature. The rotational temperature is obtained from the ratio of $(1,1)$ and $(2,2)$ column densities,

$$
T_{\text {rot }}=\frac{\left(E_{22}-E_{11}\right) / k}{\ln \left(\frac{g_{22}}{g_{11}} \frac{N(1,1)}{N(2,2)}\right)},
$$

where $E_{11}, E_{22}$, and $g_{11}, g_{22}$, are the energies and degeneracies of the corresponding levels. In practical units the equation 
Table 3

Degeneracies and Energies Above the $(1,1)$ Level of the Lower Metastables Levels of $\mathrm{NH}_{3}$ (Poynter \& Kakar 1975; Mangum \& Shirley 2015)

\begin{tabular}{lcc}
\hline \hline$(J, K)$ & $g_{J K}$ & $\left(E_{J K}-E_{11}\right) / k$ \\
\hline$(0,0)$ & $1 / 3$ & $(\mathrm{~K})$ \\
$(1,1)$ & 1 & -22.64 \\
$(2,2)$ & $5 / 3$ & 0.00 \\
$(3,3)$ & $14 / 3$ & 40.99 \\
\hline
\end{tabular}

Note. Values of the energies reported by these authors are slightly different from those given in Ho \& Townes (1983).

becomes (see Table 3),

$$
\left[\frac{T_{\mathrm{rot}}}{\mathrm{K}}\right]=\frac{40.99}{\ln \left(\frac{5}{3} \frac{N(1,1)}{N(2,2)}\right)}
$$

$\mathbf{N H}_{\mathbf{3}}$ column density. The ammonia total column density is usually estimated assuming that the population ratios between pairs of levels are given by the same rotational temperature (CTEX approximation) and that only the metastable levels $J=K$, up to $(J, K)=(3,3)$, are populated. While this assumption is reasonable for low-mass dense cores with moderate temperatures, it is not appropriate for hot cores, where $\mathrm{NH}_{3}$ inversion transitions $(6,6)$ to $(14,14)$ are detected (for instance, see Goddi et al. 2015).

In addition, since the $\mathrm{NH}_{3}(1,1)$ and $(2,2)$ levels correspond to para- $\mathrm{NH}_{3}$, no information on the ortho- $\mathrm{NH}_{3}$ is obtained from the $(1,1)$ and $(2,2)$ spectra. Thus, an ortho-to-para ratio has to be assumed. The usual assumption is to take an ortho-to-para ratio of 1 , although a value of $\sim 0.7$ has been observed in some star-forming regions (for instance, see the discussion in Faure et al. 2013). We will assume an ortho-to-para ratio of 1 .

With the former assumptions,

$$
N\left(\mathrm{NH}_{3}\right)=N(1,1) Q,
$$

with the partition function $Q$ given by

$$
Q=\sum_{J, K=0}^{3} \frac{g_{J K}}{g_{11}} e^{\left(E_{11}-E_{J K}\right) / k T_{\mathrm{rot}}} .
$$

In practical units (see Table 3 ),

$$
\begin{aligned}
N\left(\mathrm{NH}_{3}\right)= & N(1,1)\left[\frac{1}{3} e^{22.64 / T_{\mathrm{rot}}}+1\right. \\
& \left.+\frac{5}{3} e^{-40.99 / T_{\mathrm{rot}}}+\frac{14}{3} e^{-99.76 / T_{\mathrm{rot}}}\right] .
\end{aligned}
$$

Kinetic temperature. The kinetic temperature can be taken to be equal to the rotational temperature $T_{\text {rot }}$, but a better estimation can be given taking into account collisional transitions to other levels. A three-level approximation

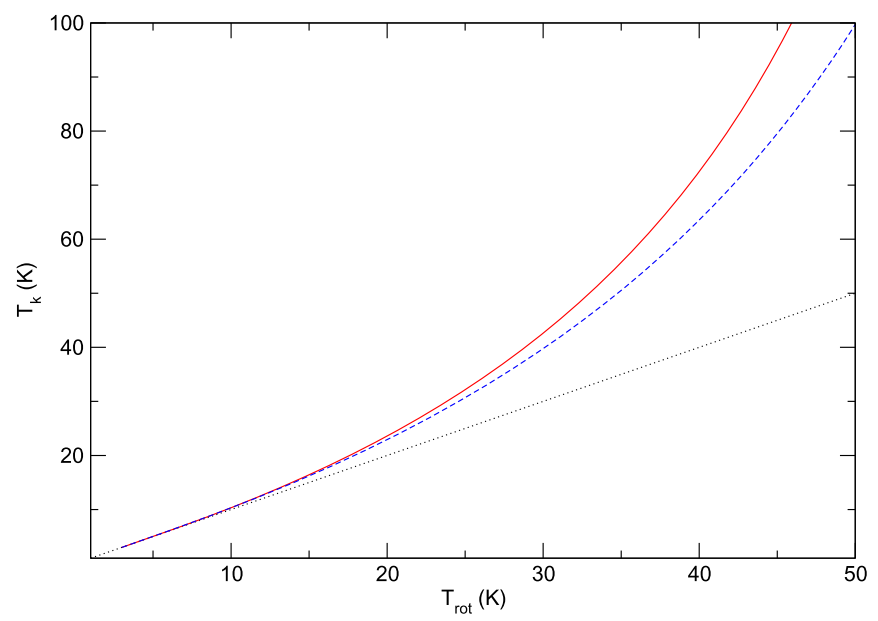

Figure 1. $T_{k}$ as a function of $T_{\text {rot }}$. Red continuum line: Maret et al. (2009) and present work; blue dashed line: Swift et al. (2005), Rosolowsky et al. (2008), Mangum \& Shirley (2015); black dotted line: $T_{k}=T_{\text {rot }}$.

(A color version of this figure is available in the online journal.)

considering only the rotational levels $(1,1),(2,2)$, and $(2,1)$ can be shown to be (Walmsley \& Ungerechts 1983; Danby et al. 1988; Mangum \& Shirley 2015)

$$
T_{\text {rot }}=\frac{T_{k}}{1+\frac{T_{k}}{T_{0}} \ln \left(1+\frac{C(22 \rightarrow 21)}{C(22 \rightarrow 11}\right)},
$$

where $T_{0}=\left(E_{22}-E_{11}\right) / k$, and $C(22 \rightarrow 21)$ and $C(22 \rightarrow 11)$ are the collisional excitation and desexcitation rates between the corresponding levels. Calculations involving more rotational levels were performed by Danby et al. (1988) and more recently by Maret et al. (2009) using improved values of the collisional rates. A good approximation to the results of Maret et al. (2009) can be given as a slightly modified version of the widely used expression (Swift et al. 2005; Rosolowsky et al. 2008; Mangum \& Shirley 2015, see Figure 1), which is

$$
T_{\text {rot }}=\frac{T_{k}}{1+\frac{T_{k}}{T_{0}} \ln \left[1+0.73 e^{-\left(E_{21}-E_{22}\right) / k T_{k}}\right]},
$$

with $T_{0}=40.99 \mathrm{~K},\left(E_{21}-E_{22}\right) / k=16.26 \mathrm{~K}$, and the numerical value 0.73 was determined to fit the results shown in Figure 5 of Maret et al. (2009). Note that this relation implies that $T_{\text {rot }}$ is always below a value $T_{\text {rot }}=40.99 / \ln 1.73=74.8 \mathrm{~K}$ (see Figure 1). Given a value of $T_{\text {rot }}$, the implicit equation must be solved to find $T_{k}$. A possible iterative algorithm to solve the equation is

$$
T_{k}^{(n+1)}=T_{\mathrm{rot}}\left[1+\frac{T_{k}^{(n)}}{40.99} \ln \left(1+0.73 e^{-16.26 / T_{k}^{(n)}}\right)\right],
$$

starting with $T_{k}^{(0)}=T_{\text {rot }}$. 


\section{Error Estimation of the Fit Parameters}

\subsection{Parameter-space Confidence Region}

Let us assume that the best fit to the observed data is obtained for values $p_{k}^{0}$ of the parameters, for which the residual $\chi^{2}$ is minimum,

$$
\chi_{\min }^{2}=\sum_{i=1}^{N}\left[\frac{y_{i}^{\mathrm{obs}}-y_{i}^{\mathrm{mod}}\left(p_{1}^{0}, \ldots, p_{m}^{0}\right)}{\sigma_{i}}\right]^{2},
$$

where $\sigma_{i}$ is the error of $y_{i}^{\text {obs }}$. The uncertainty, $\sigma\left(p_{k}\right)$, in the values derived for the parameters $p_{k}$ can be estimated as the projection of the confidence region of the $m$-dimensional space parameter for which $\chi^{2}$ does not exceed the minimum value by an amount $\Delta(m, \alpha)$, where $\alpha$ is the significance level $(0<\alpha<1)$. Following Avni (1976) and Wall \& Jenkins (2003), the probability

$$
\operatorname{Prob}\left[\chi^{2}-\chi_{\min }^{2} \leqslant \Delta(m, \alpha)\right]=\alpha,
$$

is that of a chi-square distribution with $m$ degrees of freedom. Thus, $\Delta(m, \alpha)$ is the increment of $\chi^{2}$ such that if the observation is repeated a large number of times, a fraction $\alpha$ of times the values of the parameters fitted will be inside the confidence region, i.e., in the interval $p_{k} \pm \sigma\left(p_{k}\right)$. The values of $\Delta(m, \alpha)$ for significance levels equivalents to one, two, and three sigmas for a Gaussian error distribution, and different values of $m$ are shown in Table 4 .

Assuming that the model fits well the observations, i.e., $\chi_{\min }^{2} \simeq N-m$, the condition

$$
\chi^{2}=\chi_{\min }^{2}+\Delta(m, \alpha),
$$

can be written as

$$
\frac{\chi^{2}}{\chi_{\min }^{2}} \simeq 1+\frac{\Delta(m, \alpha)}{N-m} .
$$

This expression is useful, since it can be given in terms of the weighted rms fit residual, $\sigma$,

$$
\sigma^{2}=\frac{\sum_{i=1}^{N}\left[\left(y_{i}^{\mathrm{obs}}-y_{i}^{\mathrm{mod}}\right) / \sigma_{i}\right]^{2}}{\sum_{i=1}^{N} 1 / \sigma_{i}^{2}}=\frac{\chi^{2}}{\sum_{i=1}^{N} 1 / \sigma_{i}^{2}},
$$

for which we obtain that the confidence region is given by the parameter values that increase the rms fit residual to

$$
\sigma \simeq \sigma_{\min } \sqrt{1+\frac{\Delta(m, \alpha)}{N-m}}
$$

This last expression can be used even when the errors of the observations are unknown.
Table 4

Values of $\Delta(m, \alpha)$ for Calculating the Parameter Uncertainties, where $m$ is the Number of Parameters Fitted Simultaneously, and $\alpha$ is the Significance Level, Given in Percent and in the Equivalent Number of Sigmas for a Gaussian Error Distribution. The Values shown are for Values of $m$ Multiple of 4 or 6 , Used by $H f S$

\begin{tabular}{lccc}
\hline \hline & \multicolumn{3}{c}{$\alpha$} \\
\cline { 2 - 4 }$m$ & $68.27 \%$ & $95.45 \%$ & $99.73 \%$ \\
\hline 4 & $(1 \sigma)$ & $(2 \sigma)$ & $(3 \sigma)$ \\
6 & 4.72 & 9.72 & 16.25 \\
8 & 7.04 & 12.85 & 20.06 \\
12 & 9.30 & 15.79 & 23.57 \\
16 & 13.74 & 21.35 & 30.09 \\
18 & 18.11 & 26.65 & 36.21 \\
20 & 20.28 & 29.24 & 39.17 \\
24 & 22.44 & 31.80 & 42.08 \\
28 & 26.73 & 36.83 & 47.76 \\
30 & 31.00 & 41.78 & 53.31 \\
32 & 33.12 & 44.22 & 56.04 \\
36 & 35.24 & 46.66 & 58.75 \\
42 & 39.48 & 51.48 & 64.10 \\
48 & 45.80 & 58.64 & 71.99 \\
54 & 52.11 & 65.72 & 79.75 \\
& 58.39 & 72.72 & 87.41 \\
\hline
\end{tabular}

\subsection{Modeling the Fit Residual $\chi^{2}$}

\subsubsection{Quadratic Approximation of the Fit Residual}

Let us assume that around its minimum value, the residual $\chi^{2}$ can be approximated by a quadratic function,

$$
\chi^{2} \simeq \chi_{\min }^{2}+\sum_{i, j=1}^{m} a_{i j} x_{i} x_{j}+2 \sum_{i=1}^{m} b_{i} x_{i}
$$

where $x_{j}=p_{j}-p_{j}^{0}$ are the increment of the parameter values from their best-fit values. The confidence region of the $m$ dimensional parameter space will be the region inside the surface

$$
\sum_{i, j=1}^{m} a_{i j} x_{i} x_{j}+2 \sum_{i=1}^{m} b_{i} x_{i}=\Delta(m, \alpha)
$$

which is the general equation of a $m$-dimensional quadric (McConnell 2011). This equation depends on a total of $m(m+3) / 2$ coefficients: $m(m+1) / 2$ symmetric $a_{i j}$ coefficients $\left(a_{i j}=a_{j i}\right)$ and $m$ coefficients $b_{i}$.

In array form, the quadric equation can be expressed as

$$
\left(\begin{array}{llll}
x_{1} & \ldots & x_{m} & 1
\end{array}\right)\left(\begin{array}{cccc}
a_{11} & \ldots & a_{1 m} & b_{1} \\
\vdots & \ddots & \vdots & \vdots \\
a_{m 1} & \ldots & a_{m m} & b_{m} \\
b_{1} & \ldots & b_{m} & c
\end{array}\right)\left(\begin{array}{c}
x_{1} \\
\vdots \\
x_{m} \\
1
\end{array}\right)=0
$$


with $c=-\Delta(m, \alpha)$, or with the obvious definitions for arrays $\mathbf{A}, \mathbf{B}$, and $\mathbf{X}$,

$$
\left(\begin{array}{ll}
\mathbf{X}^{t} & 1
\end{array}\right)\left(\begin{array}{cc}
\mathbf{A} & \mathbf{B} \\
\mathbf{B}^{t} & c
\end{array}\right)\left(\begin{array}{c}
\mathbf{X} \\
1
\end{array}\right)=0
$$

To estimate the uncertainties of the fit parameters, $\sigma\left(p_{k}\right)$, we have to calculate the projections of the quadric onto each axis $k$. If the fit residual is well behaved, we may expect that the quadric is an ellipsoid, i.e., its projections onto the plane defined by any pair of parameters is an ellipse and the ellipsoid has finite projections onto any axis.

\subsubsection{Projections of an Ellipsoid}

The projections of the ellipsoid onto each coordinate axis can be found as the intersections of the hyperplane perpendicular to the axis, tangent to the ellipsoid. The equation of the tangent hyperplane at a point $\left(x_{1}^{0}, \ldots, x_{m}^{0}\right)$ of the ellipsoid is given by (McConnell 2011):

$$
\left(\begin{array}{llll}
x_{1}^{0} & \ldots & x_{m}^{0} & 1
\end{array}\right)\left(\begin{array}{cccc}
a_{11} & \ldots & a_{1 m} & b_{1} \\
\vdots & \ddots & \vdots & \vdots \\
a_{m 1} & \ldots & a_{m m} & b_{m} \\
b_{1} & \ldots & b_{m} & c
\end{array}\right)\left(\begin{array}{c}
x_{1} \\
\vdots \\
x_{m} \\
1
\end{array}\right)=0
$$

or

$$
\sum_{i, j=1}^{m} a_{i j} x_{i}^{0} x_{j}+\sum_{j=1}^{m} b_{j} x_{j}+\sum_{i=1}^{m} b_{i} x_{i}^{0}+c=0 .
$$

The equation of an hyperplane perpendicular to the $k$ axis is $x_{k}$ $=$ constant so that the equation of the tangent hyperplane is

$$
\left[\sum_{i=1}^{m} a_{i k} x_{i}^{0}+b_{k}\right] x_{k}+\sum_{i=1}^{m} b_{i} x_{i}^{0}+c=0,
$$

and the coefficients of $x_{j}$ for $j \neq k$ have to be zero,

$$
\sum_{i=1}^{m} a_{i j} x_{i}^{0}+b_{j}=0 \quad(j \neq k) .
$$

Equation (45) form a system of $m-1$ linear equations with $m$ unknowns, the coordinates of the tangent point. We can consider that $x_{j}^{0}(j \neq k)$ are the $m-1$ unknowns of the system, which can be derived as a function of $x_{k}^{0}$. The system of equations can be written with arrays of size $m$,

$$
\mathbf{A}_{k} \mathbf{X}=\mathbf{Z}_{k} x_{k}^{0}+\mathbf{C}_{k},
$$

where $\mathbf{A}_{k}$ is the array $\mathbf{A}$ with zeros in the $k$ row and column, $a_{i k}=a_{k i}=0 ; 1$ in the diagonal term, $a_{k k}=1$; and $\mathbf{Z}_{k}$ and $\mathbf{B}_{k}$ have 1 and 0 , respectively, in the $k$ row:

$$
\begin{aligned}
& \mathbf{A}_{k}=\left(\begin{array}{ccccc}
a_{11} & \ldots & 0 & \ldots & a_{1 m} \\
\vdots & & \vdots & & \vdots \\
0 & \ldots & 1 & \ldots & 0 \\
\vdots & & \vdots & & \vdots \\
a_{m 1} & \ldots & 0 & \ldots & a_{m m}
\end{array}\right) \\
& \mathbf{Z}_{k}=\left(\begin{array}{c}
-a_{1 k} \\
\vdots \\
1 \\
\vdots \\
-a_{m k}
\end{array}\right), \\
&
\end{aligned}
$$

From the system, we can derive the solution giving $x_{j}^{0}$ in terms of $x_{k}^{0}$,

$$
\mathbf{X}=\mathbf{D}_{k} x_{k}^{0}+\mathbf{E}_{k}, \quad \text { with } \quad \mathbf{D}_{k}=\mathbf{A}_{k}^{-1} \mathbf{Z}_{k}, \quad \mathbf{E}_{k}=\mathbf{A}_{k}^{-1} \mathbf{C}_{k},
$$

which can be expressed as

$$
x_{j}^{0}=d_{j k} x_{k}^{0}+e_{j k} \quad(j=1, \ldots, m),
$$

where $d_{k k}=1$ and $e_{k k}=0$.

The tangent point must fulfill the tangent hyperplane equation, Equation (44). By substitution of Equation (49) in the tangent hyperplane equation and setting $x_{k}=x_{k}^{0}$, we get a second degree equation in $x_{k}^{0}$,

$$
\begin{aligned}
& {\left[\sum_{i=1}^{m} a_{k i} d_{i k}\right]\left(x_{k}^{0}\right)^{2}+\left[\sum_{i=1}^{m}\left(a_{k i} e_{i k}+b_{i} d_{i k}\right)+b_{k}\right] x_{k}^{0}} \\
& \quad+\left[\sum_{i=1}^{m} b_{i} e_{i k}+c\right]=0 .
\end{aligned}
$$

The two solutions of the equation provide the two $k$ coordinates of the projections of the ellipsoid onto the $k$ axis.

\subsubsection{Case of a Centered Ellipsoid}

Since we are assuming that we know that the minimum residual $\chi^{2}$ is well determined, the quadratic function of Equation (51) must have a minimum at the origin, and the linear terms in the variables vanish because the partial derivatives at the origin must be zero. This means that we can assume that the quadric is centered, and its equation becomes

$$
\sum_{i, j=1}^{m} a_{i j} x_{i} x_{j}+c=0 .
$$

In this case, the equation of the tangent plane is simpler, since $\mathbf{B}=0, \mathbf{C}_{k}=0$, and $\mathbf{E}_{k}=0$. The second degree equation (Equation (50)) becomes

$$
\left[\sum_{i=1}^{m} a_{k i} d_{i k}\right]\left(x_{k}^{0}\right)^{2}+c=0,
$$


and the projections are symmetric, $\pm x_{k}^{0}$.

\subsubsection{Coefficients of the Ellipsoid}

The centered ellipsoid depends on $m(m+1) / 2$ symmetric $a_{i j}$ coefficients. Let us examine a sufficient number of constraints to derive these coefficients.

Diagonal coefficients $a_{i i}$. For each parameter $i$, we can obtain constraints from the values of the residual $\chi^{2}$ increment for different increments $x_{i}$ of the parameter $p_{i}$, while keeping the rest of parameters constant,

$$
\chi^{2}\left(p_{1}^{0}, \ldots, p_{i}^{0}+x_{i}, \ldots, p_{m}^{0}\right)=\chi_{\min }^{2}+\Delta_{i} .
$$

For each value of the increments of the parameter and the residual $\chi^{2}$ increment, $x_{i}^{n}, \Delta_{i}^{n}$, we have a constraint on $a_{i i}$,

$$
a_{i i}\left(x_{i}^{n}\right)^{2} \simeq \Delta_{i}^{n} .
$$

Although a single value is enough to determine $a_{i i}$, at least two are recommended, above and below the best-fit value, i.e., with $x_{i}>0$ and $x_{i}<0$. In general, the best approximation for $a_{i i}$ (so that the sum of the squares of $\left[\Delta_{i}^{n}-a_{i i}\left(x_{i}^{n}\right)^{2}\right]$ is minimum) is given by

$$
a_{i i}=\frac{\sum_{n} \Delta_{i}^{n}\left(x_{i}^{n}\right)^{2}}{\sum_{n}\left(x_{i}^{n}\right)^{4}},
$$

where the sums are for all increments evaluated. Note that provided that the $\Delta_{i}^{n}$ are positive, the diagonal term $a_{i i}$ will always be positive. For a good characterization of the behavior of $\chi^{2}$, at least two values of $\Delta_{i}^{n}$ should be close to $\Delta(m, \alpha)$.

The intersections of the ellipsoid with the coordinate axis $i$ are $\pm\left(\Delta(m, \alpha) / a_{i i}\right)^{1 / 2}$. For the case of statistically independent parameters, these intersections will coincide with the projections of the ellipsoid, since the coordinate axes are the principal axes of the ellipsoid and the cross terms of the ellipsoid vanish. However, in general, there will be some dependence among the parameters, and the cross terms will not be zero.

Cross-coefficients $a_{i j}(i \neq j)$. The constraints to derive the cross terms $a_{i j}$ can be obtained from the value of the residual $\chi^{2}$ for the simultaneous increment of the two parameters $i$ and $j$, while keeping the rest of parameters constant,

$$
\chi^{2}\left(p_{1}^{0}, \ldots, p_{i}^{0}+x_{i}, \ldots, p_{j}^{0}+x_{j}, \ldots, p_{m}^{0}\right)=\chi_{\min }^{2}+\Delta_{i j}
$$

For each pair of increments of the parameters and the residual $\chi^{2}$ increment, $x_{i}^{n}, x_{j}^{n}, \Delta_{i j}^{n}$, we have a constraint on $a_{i j}$,

$$
a_{i i}\left(x_{i}^{n}\right)^{2}+2 a_{i j} x_{i}^{n} x_{j}^{n}+a_{j j}\left(x_{j}^{n}\right)^{2} \simeq \Delta_{i j}^{n} .
$$

Taking into account Equation (54), and defining $\delta_{i j}^{n} \equiv \Delta_{i j}^{n}-\Delta_{i}^{n}-\Delta_{j}^{n}$, we have

$$
2 a_{i j} x_{i}^{n} x_{j}^{n} \simeq \delta_{i j}^{n}
$$

Although a single pair of values $x_{i}, x_{j}$, is enough to determine $a_{i j}$, at least four are recommended, above and below the best-fit value for each parameter, i.e., with the four combinations of $x_{i}>0, x_{i}<0, x_{j}>0$, and $x_{j}<0$. In general, the best approximation for $a_{i j}$ (in the same sense as for the diagonal term) is given by

$$
a_{i j}=\frac{1}{2} \frac{\sum_{n} \delta_{i j}^{n} x_{i}^{n} x_{j}^{n}}{\sum_{n}\left(x_{i}^{n} x_{j}^{n}\right)^{2}}
$$

where the sums are for all the pairs of increments evaluated.

\subsubsection{Practical Case}

A practical implementation of the procedure is as follows. Let us call

$$
f\left(x_{1}, \ldots, x_{m}\right)=\chi^{2}\left(p_{1}^{0}+x_{1}, \ldots, p_{m}^{0}+x_{m}\right)-\chi_{\min }^{2} .
$$

1. For each parameter $p_{i}$, we estimate a positive and a negative increment, $x_{i}^{+}$and $x_{i}^{-}$, such that the increment of the residual $\chi^{2}$ is close to $\Delta(m, \alpha)$,

$$
\begin{aligned}
& f\left(0, \ldots, x_{i}^{+}, \ldots, 0\right)=\Delta_{i}^{+} \simeq \Delta(m, \alpha), \\
& f\left(0, \ldots, x_{i}^{-}, \ldots, 0\right)=\Delta_{i}^{-} \simeq \Delta(m, \alpha) .
\end{aligned}
$$

The diagonal coefficient $a_{i i}$ (Equation (54)) is given by

$$
a_{i i}=\frac{\Delta_{i}^{+}\left(x_{i}^{+}\right)^{2}+\Delta_{i}^{-}\left(x_{i}^{-}\right)^{2}}{\left(x_{i}^{+}\right)^{4}+\left(x_{i}^{-}\right)^{4}} .
$$

2. For each pair of parameters $p_{i}, p_{j}(i \neq j)$ we calculate

$$
\begin{aligned}
& \delta_{i j}^{++}=f\left(0, \ldots, x_{i}^{+}, \ldots, x_{j}^{+}, \ldots, 0\right)-\Delta_{i}^{+}-\Delta_{j}^{+}, \\
& \delta_{i j}^{+-}=f\left(0, \ldots, x_{i}^{+}, \ldots, x_{j}^{-}, \ldots, 0\right)-\Delta_{i}^{+}-\Delta_{j}^{-}, \\
& \delta_{i j}^{-+}=f\left(0, \ldots, x_{i}^{-}, \ldots, x_{j}^{+}, \ldots, 0\right)-\Delta_{i}^{-}-\Delta_{j}^{+}, \\
& \delta_{i j}^{--}=f\left(0, \ldots, x_{i}^{-}, \ldots, x_{j}^{-}, \ldots, 0\right)-\Delta_{i}^{-}-\Delta_{j}^{-} .
\end{aligned}
$$

The cross-coefficient $a_{i j}$ (Equation (59)) is given by

$$
a_{i j}=\frac{1}{2} \frac{\delta_{i j}^{++} x_{i}^{+} x_{j}^{+}+\delta_{i j}^{+-} x_{i}^{+} x_{j}^{-}+\delta_{i j}^{-+} x_{i}^{-} x_{j}^{+}+\delta_{i j}^{--} x_{i}^{-} x_{j}^{-}}{\left(x_{i}^{+} x_{j}^{+}\right)^{2}+\left(x_{i}^{+} x_{j}^{-}\right)^{2}+\left(x_{i}^{-} x_{j}^{+}\right)^{2}+\left(x_{i}^{-} x_{j}^{-}\right)^{2}} .
$$

3. For each parameter $k$, we construct the arrays $\mathbf{A}_{k}, \mathbf{Z}_{k}$ (Equation (47)) and solve the system of linear equations $\mathbf{A}_{k} \mathbf{D}_{k}=\mathbf{Z}_{k}$. Finally, we calculate the quadratic coefficient, $\sum_{i=1}^{m} a_{k i} d_{i k}$ (Equation (52)) and the projection $x_{k}^{0}=\left[\Delta(m, \alpha) / \sum_{i=1}^{m} a_{k i} d_{i k}\right]^{1 / 2}$.

In some cases, the quadratic approximation of $\chi^{2}$ is not good enough and the determination of the projections can fail: for some parameter $p_{k}$, the array $\mathbf{A}_{k}$ may have null determinant or the quadratic coefficient of Equation (52) may be negative. In these cases, a rough estimation of the uncertainty in $p_{k}$ can still be given as the intersection with the $k$ axis, $\left[\Delta(m, \alpha) / a_{k k}\right]^{1 / 2}$. 
Table 5

Comparison of the Results Obtained with $H f S$ and CLASS for a Sample of $\mathrm{NH}_{3}(1,1)$ Spectra

\begin{tabular}{|c|c|c|c|c|c|c|c|}
\hline Id. $^{\text {a }}$ & $\begin{array}{c}\Delta V \\
\left(\mathrm{~km} \mathrm{~s}^{-1}\right)\end{array}$ & $\begin{array}{c}V_{\mathrm{LSR}} \\
\left(\mathrm{km} \mathrm{s}^{-1}\right)\end{array}$ & $A\left(1-\exp _{(\mathrm{K})}\left\{-\tau_{m}\right\}\right)$ & $1-\exp \left\{-\tau_{m}\right\}$ & $\begin{array}{l}A \tau_{m} \\
(\mathrm{~K})\end{array}$ & $\tau_{m}$ & Rout. $^{b}$ \\
\hline \multirow[t]{2}{*}{$\overline{1}$} & $1.221 \pm 0.194$ & $-3.029 \pm 0.061$ & $6.2 \pm 0.8$ & $0.2 \pm 0.5$ & $7.1 \pm 3.5$ & $0.3 \pm 0.9$ & $H f S$ \\
\hline & $1.240 \pm 0.091$ & $-3.030 \pm 0.027$ & $\ldots$ & $\ldots$ & $7.1 \pm 1.0$ & $0.3 \pm 0.3$ & $\mathrm{C}$ \\
\hline & $0.750 \pm 0.007$ & $-4.550 \pm 0.003$ & $\ldots$ & $\ldots$ & $85.9 \pm 1.6$ & $5.54 \pm 0.13$ & $\mathrm{C}$ \\
\hline \multirow[t]{2}{*}{3} & $0.569 \pm 0.066$ & $-20.445 \pm 0.030$ & $4.5 \pm 0.4$ & $0.970 \pm 0.023$ & $16.2 \pm 4.8$ & $3.5 \pm 1.1$ & $H f S$ \\
\hline & $1.030 \pm 0.001$ & $-20.500 \pm 0.011$ & $\ldots$ & $\ldots$ & $8.0 \pm 0.4$ & $1.4 \pm 0.2$ & $\mathrm{C}$ \\
\hline \multirow[t]{2}{*}{5} & $3.753 \pm 0.358$ & $-2.180 \pm 0.168$ & $6.8 \pm 0.6$ & $0.7 \pm 0.2$ & $12.3 \pm 4.0$ & $1.3 \pm 0.8$ & $H f S$ \\
\hline & $3.410 \pm 0.039$ & $-2.160 \pm 0.022$ & $\ldots$ & $\ldots$ & $9.87 \pm 0.10$ & $0.84 \pm 0.03$ & $\mathrm{C}$ \\
\hline
\end{tabular}

Notes.

${ }^{a}$ Spectra analyzed in the comparison. 1: low optical depth; 2: very high optical depth; 3 , 4: lines narrower than the channel width $\left(0.6 \mathrm{~km} \mathrm{~s} \mathrm{~s}^{-1}\right)$, moderate and high optical depths; 5: very broad lines.

${ }^{\mathrm{b}}$ Routines being compared. HfS: present work; C: CLASS, using method NH3(1,1).

\section{Error Estimation of the Derived Parameters}

All the derived parameters depend on $m$ fit parameters (four or six times the number of velocity component). Let us call

$$
p_{k}^{0}, \quad k=1, \ldots, m
$$

the values of the fit parameters and $\sigma\left(p_{k}\right)$ their errors found from the increase in the fit residual $\chi^{2}$ (see Section 7). Let $d$ be any of the parameters derived from the fit parameters, $d=d$ $\left(p_{1}, \ldots, p_{m}\right)$, for instance $\tau_{m}$ or $N\left(\mathrm{NH}_{3}\right)$. For every fit parameter $p_{k}^{0}(k=1, \ldots, m)$, we evaluate the values of the derived parameter when we increase and decrease the value of the $k$-th fit parameter by its error $\sigma\left(p_{k}\right)$,

$$
\begin{array}{ll}
d_{k}^{+}=d\left(p_{1}^{0}, \ldots, p_{k}^{0}+\sigma\left(p_{k}\right), \ldots, p_{m}^{0}\right), & k=1, \ldots, m \\
d_{k}^{-}=d\left(p_{1}^{0}, \ldots, p_{k}^{0}-\sigma\left(p_{k}\right), \ldots, p_{m}^{0}\right), & k=1, \ldots, m
\end{array}
$$

Assuming that the errors of the fit parameters are statistically independent, we can estimate the error $\sigma(d)$ as

$$
\sigma^{2}(d)=\sum_{k=1}^{m}\left(\frac{d_{k}^{+}-d_{k}^{-}}{2}\right)^{2}
$$

\section{Comparison of $\boldsymbol{H} \boldsymbol{f S}$ with Other Routines}

The fits obtained with $H f S$ and $H f S \_n h 3$ were compared with those obtained with other commonly used routines. In Tables 5 and 6, we show some examples of fits performed with $H f S$ and CLASS for the $\mathrm{NH}_{3}(1,1)$ line and the Rosolowsky routine (Rosolowsky et al. 2008) for the simultaneous fit of the $\mathrm{NH}_{3}(1$, $1)$ and $(2,2)$ lines. The fits shown cover cases of low and high optical depth, and narrow and wide linewidths. As can be seen in the tables, there is in general agreement between the $H f S$ results and the other routines.
Regarding the comparison with CLASS, in the case of linewidths lower than the channel width (spectra 3 and 4 in Table 5), CLASS gives a fixed value for the linewidth, higher than the channel width and much higher than the actual linewidth. As a consequence, the values of $A \tau_{m}$ and $\tau_{m}$ given by CLASS are scaled down by roughly the same factor so that CLASS is consistent with $H f S$ in the values of $A \tau_{m} \Delta V$ and $\tau_{m} \Delta V$. In general, the errors given by CLASS appear to be underestimated.

The comparison with the Rosolowsky routine was performed for the examples of fits given in Rosolowsky et al. (2008). The fitted parameters were taken from Table 3 of the electronic edition of Rosolowsky et al. (2008), and the raw spectral data were retrieved from the COMPLETE Web site. ${ }^{5}$ For the $H f S \_n h 3$ fits, the data were Hanning-smoothed with HFHW $=2$ and fitted using Nksample $=400$ (see below). For the parameters obtained specifically from the simultaneous fit of the $\mathrm{NH}_{3}(1,1)$ and $(2,2)$ lines, there is a good agreement in the values obtained for $T_{k}$, and the values of ammonia column density reported in Rosolowsky et al. (2008) lie between the two limiting cases given by $H f S \_n h 3$, for filling factors $f \ll 1$ and $f=1$.

\section{Description of the $H f S$ Procedures}

While the $H f S \_n h 3$ procedures fit the $\mathrm{NH}_{3}(1,1)$ and $(2,2)$ transitions simultaneously, the general $H f S$ procedures fit a single transition, selected among those stored in the file hfs_transitions.dat. This file has to be located in the working directory or in the directory pointed at by the environment variable HFS_DIR (see Appendix A). The first transition in the file is a single line at $V_{\mathrm{LSR}}=0$, useful for

\footnotetext{
5 https://www.cfa.harvard.edu/COMPLETE/data_html_pages/GBT_ NH3.html
} 
Table 6

Comparison of the Results Obtained with $H f S$ and the Routines of Rosolowsky et al. $(2008)$ for a Sample of $\mathrm{NH}_{3}(1,1)$ and $(2,2)$ Spectra

\begin{tabular}{|c|c|c|c|c|c|c|c|c|c|}
\hline \multirow[b]{3}{*}{$\mathrm{Id}^{\mathrm{c}}$} & \multirow{3}{*}{$\begin{array}{c}\Delta V^{\mathrm{b}} \\
\left(\mathrm{km} \mathrm{s}^{-1}\right)\end{array}$} & \multirow{3}{*}{$\begin{array}{c}V_{\mathrm{LSR}} \\
\left(\mathrm{km} \mathrm{s}^{-1}\right)\end{array}$} & \multirow{3}{*}{$\begin{array}{c}A \tau_{1 m}{ }^{\mathrm{b}} \\
(\mathrm{K})\end{array}$} & \multirow[b]{3}{*}{$\tau_{1 m}{ }^{\mathrm{b}}$} & \multirow{3}{*}{$\begin{array}{l}T_{\mathrm{ex}} \\
(\mathrm{K})\end{array}$} & \multirow{3}{*}{$\begin{array}{c}T_{k} \\
(\mathrm{~K})\end{array}$} & \multirow{2}{*}{\multicolumn{2}{|c|}{$\begin{array}{c}N\left(\mathrm{NH}_{3}\right)^{\mathrm{a}} \\
\left(10^{13} \mathrm{~cm}^{-3}\right)\end{array}$}} & \multirow[b]{3}{*}{ Rout. } \\
\hline & & & & & & & & & \\
\hline & & & & & & & $(f \ll 1)$ & $(f=1)$ & \\
\hline \multirow[t]{2}{*}{$\overline{16}$} & $0.335 \pm 0.034$ & $4.596 \pm 0.013$ & $0.90 \pm 0.12$ & $1.12 \pm 0.12$ & $3.53 \pm 0.12$ & $9.9 \pm 1.9$ & $3.8 \pm 2.1$ & $17.1 \pm 9.9$ & $H f S$ \\
\hline & $0.332 \pm 0.014$ & $4.614 \pm 0.007$ & $1.85 \pm 0.8$ & .. & $\ldots$ & $<13$. & & $>1.27$ & $\mathrm{R}$ \\
\hline \multirow[t]{2}{*}{31.1} & $0.390 \pm 0.008$ & $4.618 \pm 0.005$ & $5.67 \pm 0.12$ & $1.56 \pm 0.12$ & $6.37 \pm 0.06$ & $12.6 \pm 1.7$ & $20.3 \pm 3.5$ & $35.7 \pm 6.2$ & $H f S$ \\
\hline & $0.400 \pm 0.024$ & $4.6 \pm 0.2$ & & $1.875 \pm 0.001$ & & $11.7 \pm 0.1$ & & $23 \pm 1.0$ & $\mathrm{R}$ \\
\hline \multirow[t]{2}{*}{31.2} & $0.327 \pm 0.023$ & $5.984 \pm 0.016$ & $1.37 \pm 0.14$ & $1.52 \pm 0.14$ & $3.64 \pm 0.09$ & $10.6 \pm 2.3$ & $5.1 \pm 2.7$ & $21 \pm 11$ & $H f S$ \\
\hline & $0.306 \pm 0.047$ & $6.01 \pm 0.05$ & $\ldots$ & $0.870 \pm 0.001$ & $\ldots$ & $10.4 \pm 0.1$ & $\ldots$ & $10 \pm 1.0$ & $\mathrm{R}$ \\
\hline \multirow[t]{2}{*}{47} & $0.443 \pm 0.007$ & $8.1714 \pm 0.0020$ & $16.83 \pm 0.38$ & $4.09 \pm 0.38$ & $6.86 \pm 0.04$ & $11.49 \pm 0.30$ & $75.9 \pm 3.6$ & $126.8 \pm 5.9$ & $H f S$ \\
\hline & $0.444 \pm 0.001$ & $8.1840 \pm 0.0007$ & $\ldots$ & $4.075 \pm 0.035$ & $7.82 \pm 0.02$ & $11.69 \pm 0.04$ & $\ldots$ & $73.2 \pm 0.7$ & $\mathrm{R}$ \\
\hline \multirow[t]{2}{*}{89} & $0.294 \pm 0.006$ & $8.1617 \pm 0.0024$ & $12.00 \pm 0.35$ & $3.23 \pm 0.35$ & $6.46 \pm 0.09$ & $9.9 \pm 1.0$ & $44.6 \pm 8.4$ & $78 \pm 15$ & $H f S$ \\
\hline & $0.294 \pm 0.002$ & $8.1790 \pm 0.0010$ & $\ldots$ & $2.75 \pm 0.10$ & $7.9 \pm 0.1$ & $10.5 \pm 0.1$ & $\ldots$ & $39 \pm 1$ & $\mathrm{R}$ \\
\hline \multirow[t]{2}{*}{93} & $0.227 \pm 0.005$ & $6.002 \pm 0.003$ & $4.93 \pm 0.23$ & $2.48 \pm 0.23$ & $4.73 \pm 0.09$ & $9.9 \pm 0.9$ & $14.1 \pm 2.4$ & $33.6 \pm 5.6$ & $H f S$ \\
\hline & $0.231 \pm 0.002$ & $6.022 \pm 0.001$ & $\ldots$ & $1.65 \pm 0.10$ & $6.1 \pm 0.2$ & $10.5 \pm 0.2$ & $\ldots$ & $14 \pm 1.0$ & $\mathrm{R}$ \\
\hline \multirow[t]{2}{*}{95} & $0.343 \pm 0.003$ & $6.044 \pm 0.002$ & $21.95 \pm 0.11$ & $6.66 \pm 0.11$ & $6.04 \pm 0.02$ & $9.70 \pm 0.01$ & $98.8 \pm 0.8$ & $181.6 \pm 1.2$ & $H f S$ \\
\hline & $0.342 \pm 0.002$ & $6.0635 \pm 0.0009$ & $\ldots$ & $6.90 \pm 0.10$ & $6.75 \pm 0.02$ & $10.01 \pm 0.06$ & $\ldots$ & $106 \pm 2.0$ & $\mathrm{R}$ \\
\hline
\end{tabular}

Notes.

a Beam-averaged $\mathrm{NH}_{3}$ column densities calculated for filling factors $f \ll 1$ and $f=1$.

${ }^{\mathrm{b}}$ Calculated from the values of $\sigma_{V}$ and $\tau_{\text {tot }}$ given in Rosolowsky et al. (2008).

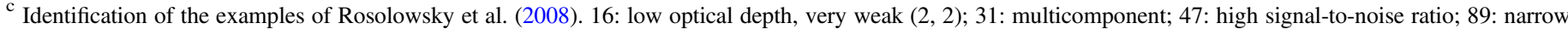
lines; 93: good fit, weak $(2,2)$; 95: high optical depth.

${ }^{\mathrm{d}}$ Routines being compared. HfS: present work; R: Rosolowsky et al. (2008).

fitting a single Gaussian line. Other transitions in the file include $\mathrm{NH}_{3}(1,1)$ and $(2,2), \mathrm{NH}_{2} \mathrm{D}\left(1_{11}, 1_{10}\right), \mathrm{N}_{2} \mathrm{H}^{+}(1-0)$, $\mathrm{CN}$, $\mathrm{HCN}, \mathrm{H}^{13} \mathrm{CN}, \mathrm{C}_{2} \mathrm{H}, \mathrm{C}_{2} \mathrm{D}, \mathrm{C}^{17} \mathrm{O}$, and more transitions can be added easily to the file. The criterion for defining the "main component" of a transition is, in general, all the hyperfines with a velocity offset less than $0.001 \mathrm{~km} \mathrm{~s}^{-1}$, and for $\mathrm{NH}_{3}(1,1)$ and $(2,2)$, less than $0.6 \mathrm{~km} \mathrm{~s}^{-1}$. Any transition not appearing in the file is assumed to be single.

An optional Hanning smoothing of the spectrum can be performed prior to fitting. You can select the Hanning filter half-width (HFHW). For $\mathrm{HFHW}=0$, no smoothing is performed. $\mathrm{HFHW}=1$ is the standard three-point Hanning smoothing, and the resulting spectrum has half the initial number of channels. In general, the Hanning smoothing encompasses $2 * H F H W+1$ points, resulting in a final number of channels $\mathrm{HFHW}+1$ times smaller.

The iterative process is controlled by two parameters: Nksample, the number of thousands of samples of the parameter space, and Final_Range, the ratio of ranges of the last loop and initial search ranges. The number of loops is taken as nloop $=$ Nksample $e^{1 / 2}$ and the number of seeds and descendants is taken as nseed $=$ ndesc $=(\text { nloop } \times 1000)^{1 / 2}$, so that nloop $\times$ nseed $\times$ ndesc $=$ Nksample $\times 1000$. For each loop the ranges will be decreased a factor of $f=$ Final_Range $\mathrm{e}^{1 /(\mathrm{nloop}-1)}$.

The initial values for the fit are guessed from the intensity, position, and width of the data peak for the first component, and of the residual (data minus previous components) for the rest of components (up to a maximum of 9). The main line optical depth is set arbitrarily to $0.7\left(1-e^{-\tau_{m}}=0.5\right)$, or to an arbitrary low value $\left(10^{-6}\right)$ for a single Gaussian fit.

Values for the initial search ranges are calculated by the procedures. For fitting a single Gaussian line, the search range of $\tau_{m}^{*}$ is made 0 to keep it constant. Additional constraints for the search ranges of the parameters are $A_{m}^{*}>0 ; 0<\tau_{m}^{*}<1$; and $\Delta V>\Delta V_{\min }$, where $\Delta V_{\min }$ is the thermal linewidth for a kinetic temperature $T_{\mathrm{k}}=T_{\mathrm{bg}}$ of a large molecule (for the general HfS procedures, $0.025 \mathrm{~km} \mathrm{~s}^{-1}$ for a mass of $200 \mathrm{~m}_{\mathrm{H}}$ ), or of $\mathrm{NH}_{3}\left(0.086 \mathrm{~km} \mathrm{~s}^{-1}\right.$ for the $H f S \_n h 3$ procedures).

Note that the final value of a fit parameter can be outside the initial range for the parameter. For an initial range $2 r$ and a range decreasing factor per loop $f$, the final value of a parameter can can be up to roughly $r /(1-f)$ apart from its initial value. For instance, for the default values Nksample $=200$ (corresponding to nloop $=14$ ) and Final_Range $=0.05$, we have $f \simeq 0.8$ and $r /(1-f) \simeq 5 r$.

The different procedures that compose $H f S$ are described in the following.

\section{1. hfs fit, hfs nh3}

These are interactive graphic procedures for fitting simultaneously multiple velocity components of an spectral line with hyperfine structure, or to a pair of $\mathrm{NH}_{3}(1,1)$ and $(2,2)$, to spectra read from data files, and generating files with the 

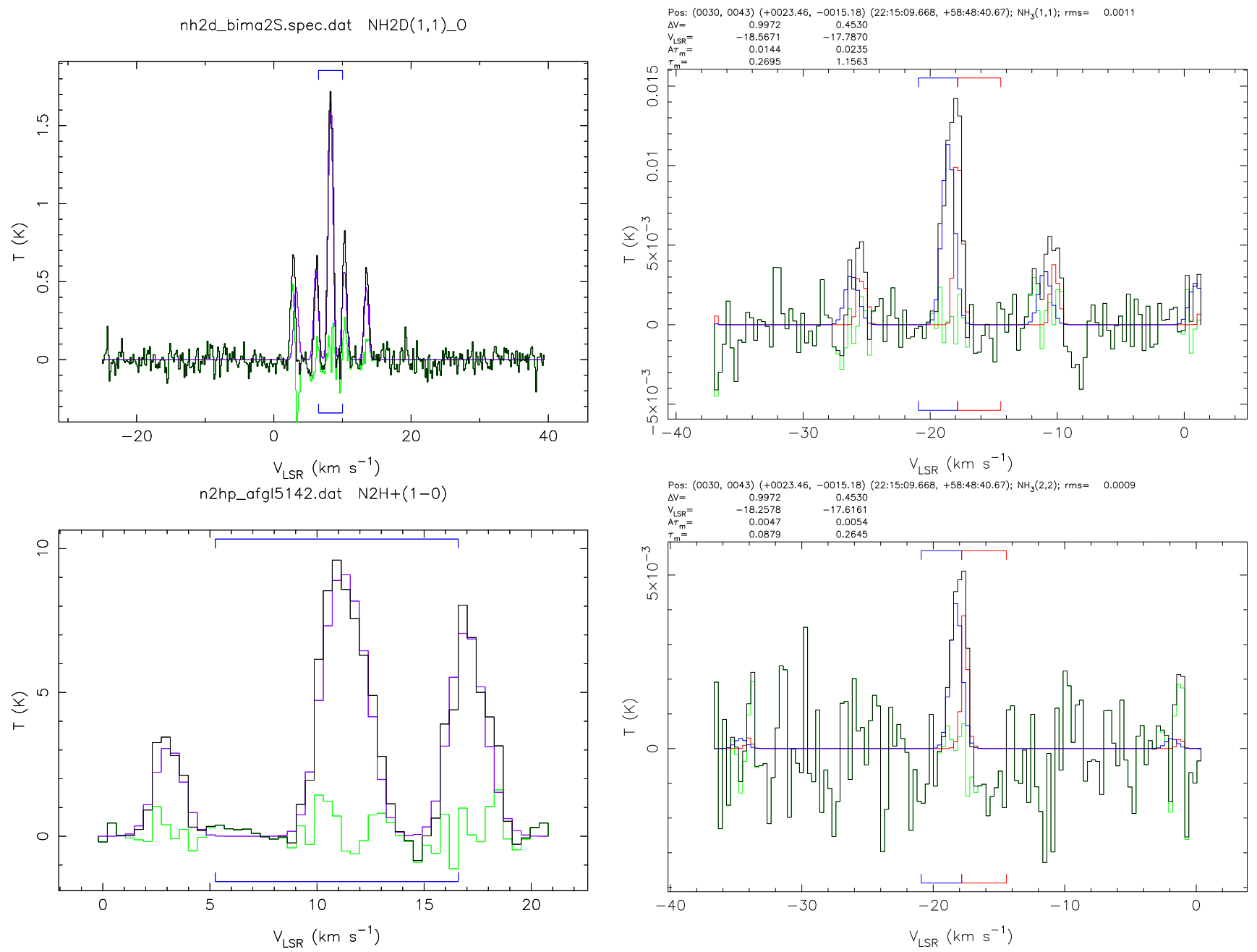

Figure 2. Left: $\mathrm{NH}_{2} \mathrm{D}(1,1)$ (top) $\mathrm{N}_{2} \mathrm{H}^{+}(1-0)$ (bottom) fits using hfs_fit. The color lines indicate the data (black), fit (magenta), and residual (green). Right: $\mathrm{NH}_{3}(1$, $1)$ and $(2,2)$ simultaneous fit of two velocity components using hfs_nh3. The blue and red lines indicate the two velocity components fitted. The search range for each component is indicated by the blue and red segments at the top and bottom of each plot.

(A color version of this figure is available in the online journal.)

synthetic spectra. See an example of a fitting run of $h f s_{-}$fit in Appendix D and of hfs_nh3 in Appendix E.

You can set any number of velocity components for which the procedures propose a first guess. Alternatively, you can use the cursor to add or delete components. The position of a new component is set at the cursor position, its intensity is the intensity at the cursor position, and the FWHM is estimated around the cursor position. The values of the initial search ranges can be changed. Any of the parameters can be kept constant by setting its range to 0 .

\section{Input}

The spectra to fit are read from ASCII files with a pair of values (velocity, intensity) per line. Lines beginning with "!" or "\#" are ignored. The file(s) can be given as argument(s) to hfs_fit and hfs_nh3:

\$hfs_fit $<$ source $>$.dat

\$hfs_nh3 < source_11 > dat < source_22 > dat

\section{Output}

- hfs_fit.log or hfs_nh3.log, log file with the details of the fitting session.

- <source>.synt, or <source_11>.synt and $<$ source_22>.synt, ASCII files with the synthesized spectra. The files have a header (lines beginning with "!") with the values of the parameters of the fitted spectra for each velocity component. The files are readable by GREG of the package GILDAS. The file is overwritten for every new fit. See an example in Appendix G. 
- <source $>$.eps, plot showing the data, the components fitted, and the residual. See some examples in Figure 2. The file is overwritten for every new fit.

\section{2. hfs_file.}

This is a batch procedure to fit the hyperfine structure of the same transition (and a single velocity component) for a set of data files and for generating a list of files with the synthetic spectra. The fit procedure is the same as that of $h f_{S} \_$fit, but it is not interactive.

\section{Input}

The input is a parameter file that can be given as argument when running the procedure:

\$hfs_file $<$ file_list $>$.par

The contents of the file is as follows:

1. Transition name, e.g., "NH3(1,1)";

2. Iteration parameters: Nksample, Final_Range; and

3. Following lines: list of files, one file per line

See an example of $<$ file_list $>$.par in Appendix F.

\section{Output}

- $<$ file_list $>$. $\log , \log$ file with the details of the fitting process for all the files in $<$ file_list $>$.par.

- $<$ file_list>.out, ASCII file with the values and uncertainties of the parameters fitted for each file of the list. The first lines beginning with "!" are the header and give information about the transition and the column headers.

- $<$ file_list $>$.ps, PostScript file with plots of the data, the components fitted, and the residual for all the files in the list.

- $<$ file_\#>. synt, an ASCII file for each file in $<$ file_list>.par, with the synthesized spectrum. The file has a header (lines beginning with "'”) with the values of the parameters of the fitted spectrum.

\section{3. hfs_cube_sp, hfs_nh3_cube_sp}

These are single-processor batch procedures for fitting simultaneously multiple velocity components of spectra from 3-axes FITS data cubes. A subimage of the FITS data cube can be selected and optional boxcar averaging of pixels and Hanning filtering of the spectra can be performed. The procedures are similar to $\mathrm{hfs}$ _fit and $\mathrm{hfs}$ _nh 3 , but they are not interactive. The maximum dimension of the image and the maximum number of channels of the data cube is arbitrary, i.e., the only limitation is the amount of memory available by the computer.

\subsubsection{Input}

The input is a parameter file that has to be given as argument when running the procedures:

\$hfs_cube_sp < parameter $>$.par

\$hfs_nh3_cube_sp < parameter $>$.par

The parameter file for hfs_cube_sp has 10 lines (see an example in Appendix F):

1. Transition name, e.g., "C170 (1-0)".

2. FITS data cube file to read. The file must have 3 nondegenerate axes, in this order: $x$ position, y position, velocity channel.

3. rms of channels without emission, minimum SNR of spectra to be fitted. Components with a peak intensity below SNR times rms are not fitted.

4. Number of velocity components to fit, Ncomp, between 1 and 9.

5. Range of channels for each component: $2 \times$ Ncomp values, with the first and last channel of the velocity range for each component. The channel ranges must be nonoverlapping. For the first component, 0 defaults to 1 (first), nchan (last).

6. HFHW (channels), 0 for no filtering.

7. Boxcar smoothing radius (pixels), 0 for no smoothing.

8. Subimage to be fitted: first $X$ pixel, last $X$ pixel, $X$ increment. 0 defaults to 1 (first), ndim1 (last), 1 (increment).

9. Subimage to be fitted: first $\mathrm{Y}$ pixel, last $\mathrm{Y}$ pixel, $\mathrm{Y}$ increment. 0 defaults to 1 (first), ndim2 (last), 1 (increment).

10. Iteration parameters: Nksample, Final_Range.

The parameter file for hfs_nh3_cube_sp has 11 lines (see an example in Appendix F):

1. $(1,1)$ FITS data cube file to read. The file must have three non-degenerate axes in this order: x position, y position, and velocity channel.

2. $(1,1)$ rms of channels without emission, minimum SNR of spectra to be fitted. Components with a peak intensity below SNR times rms are not fitted.

3. Number of velocity components to fit, Ncomp, between 1 and 9.

4. $(1,1)$ range of channels for each component: $2 \times$ Ncomp values, with the first and last channel of the velocity range for each component. The channel ranges must be 

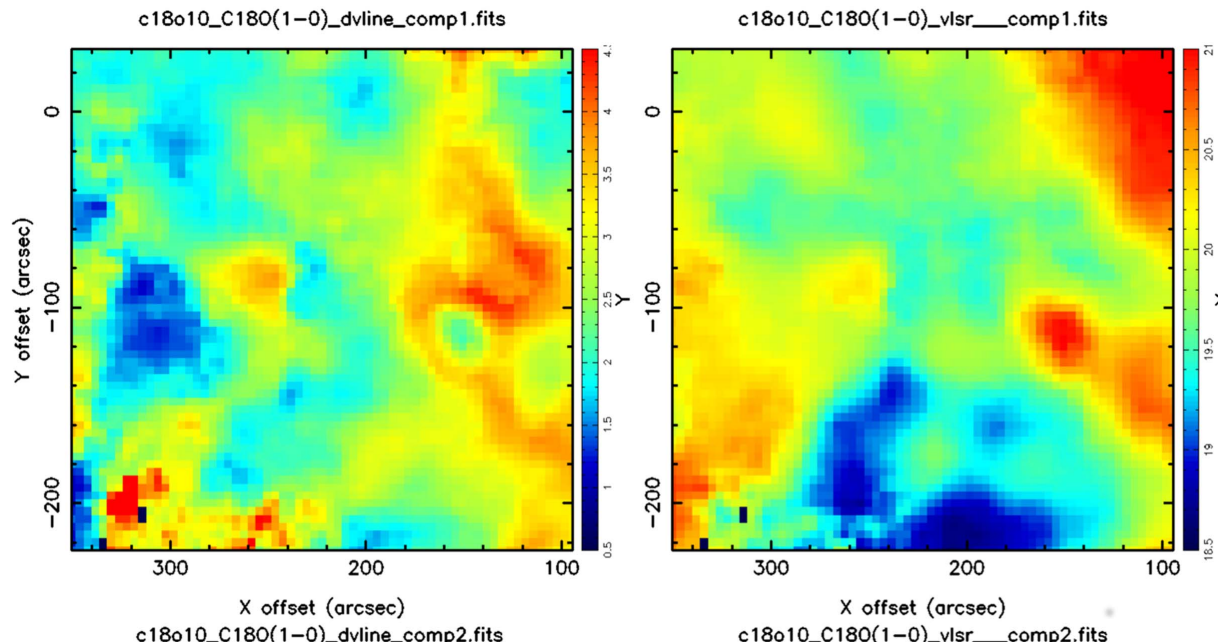

c18010_C180(1-0)_vlsr__comp2.fits
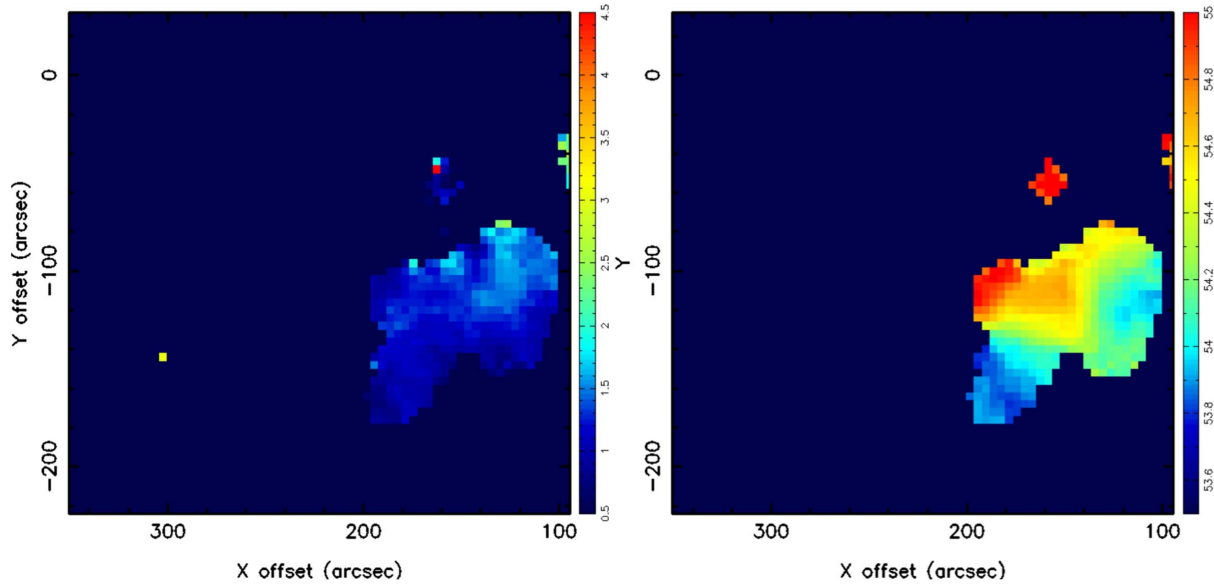

c18010_c180(1-0)_ataum comp1.fits

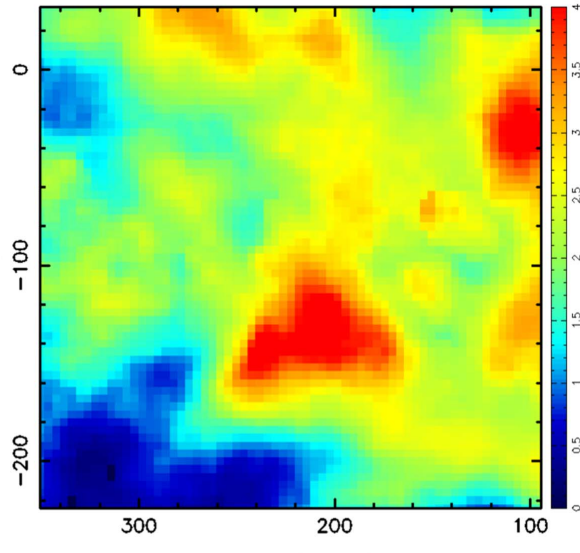

$X$ offset (arcsec)

c18010_c180(1-0)_ataum_comp2.fits

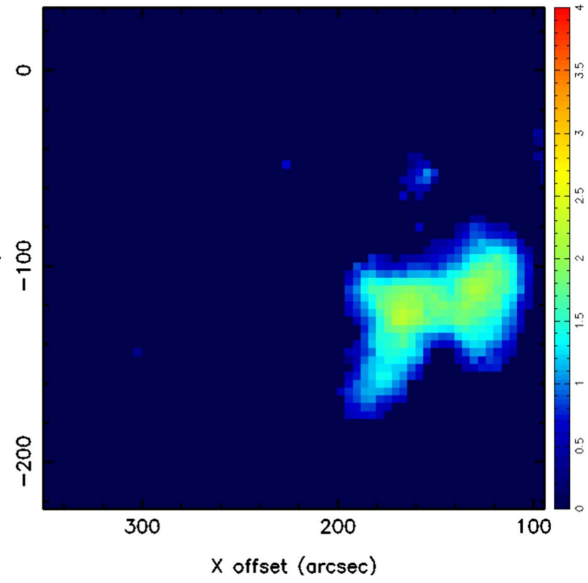

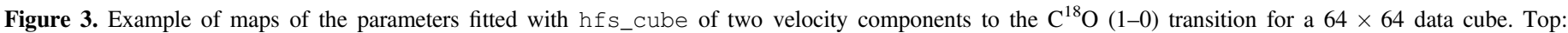
component 1; bottom: component 2, left: $\Delta V$, middle: $V_{\mathrm{LSR}}$, right: $A \tau_{m}$.

(A color version of this figure is available in the online journal.)

non-overlapping. For the first component, 0 defaults to 1 (first), nchan (last).

5. $(2,2)$ FITS data cube file to read. The file must have the same geometry as that of the $(1,1)$ FITS file.

6. $(2,2)$ rms of channels without emission, minimum SNR of spectra to be fitted. Components with a peak intensity below SNR times rms are not fitted.

The five following lines are the same as for the case of hfs_cube_sp.

\section{Output}

- $\log /<$ parfile>. log, log file in folder $\log$ with the details of the fitting process for all the pixels of the subimage.
- <parfile>_comp\# . out, an ASCII file for each velocity component with the values of the parameters fitted and the line and physical parameters for each pixel of the subimage, and their uncertainty. The first lines beginning with "!" are the header and give information about the parameter file, FITS files, velocity component number, velocity range of the component, Hanning filtering applied, smoothing boxcar radius, and column headers. The files are used by hfs_view, and are readable by GREG. See an example in Appendix G.

- ps/<parfile>_<xoffset>.ps, PostScript files in folder ps, with plots of the data, the components fitted, and the residual for all pixels of the subimage with a given $<$ xoffset $>$ in arcsec. 
- maps / <parfile>_<parameter>_comp\#.fits, FITS files in folder maps, with maps, for each velocity component, of the parameters fitted and the line and physical parameters, and their uncertainty. Each FITS file has two planes, the first plane with the values of the parameter, and the second plane with the uncertainties. See in Figure 3 an example of the the maps obtained from a 2-velocity-components fit of the $\mathrm{C}^{18} \mathrm{O}$ line for a FITS data cube.

\section{4. hfs_cube_mp, hfs_nh3_cube_mp}

These are multiprocessor procedures that use Open MPI (see Appendix A) and run in parallel using a number of processors available in the machine or in more than one host. The multiprocessor procedures are naked versions of the singleprocessor versions, hfs_cube_sp and hfs_nh3_cube_sp without any graphic output. The instructions for running these procedures can be found in Appendix C.

\section{5. hfs_view, hfs_nh3_view}

These are interactive graphic procedures for displaying spectra from FITS data cubes and the corresponding synthetic spectra fitted with hfs_cube or hfs_nh3_cube. A plot of the integrated intensity is shown, and you can select with the mouse the position for which the the spectrum (data and synthetic) is shown. You can select to show the integrated intensity for all channels or for the channel ranges of each velocity component. The data and synthetic spectra at any pixel can be extracted in ASCII files.

\section{Input}

The input is the same parameter file used as input to hfs_cube or hfs_nh3_cube. From the information in this file, hfs_view reads the corresponding FITS data cubes and the <parfile>_comp\#.out file for each velocity component (created by hfs_cube or hfs_nh3_cube), with the parameters of the fitted spectra.

\section{Output}

For any pixel you can extract the data and synthetic spectra:

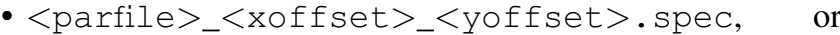
$<$ parfile $>$ 11__xoffset $\rangle_{-}<y o f f$ set $>$. spec and $\langle$ parfile $>$ 22__<offset $>$ _ $<$ yoffset $>$.spec, data spectra for the pixel selected, with the Hanning filtering and boxcar smoothing given in the $<$ parfile $>$.par file. See an example in Appendix G.

- <parfile $>_{-}<$xoffset $>_{-}<y o f f s e t>$.synt, or $<$ parfile $>$ _11_<xoffset $>$ _<yoffset $>$. synt and $<$ parfile $>$ _22_<xoffset $>$ _<yoffset $>$.synt, synthetic spectra fitted for the pixel selected.

\section{6. hfs_blanking}

This is an auxiliary procedure to flag the output FITS files of hfs_cube and hfs_nh3_cube, according to the parameter values, errors, or SNR. The FITS files have two planes: the first one with the values of the parameter at each pixel and the second one with with the error of the value. The procedure reads the values and errors in the FITS files and allows you to blank the pixels that fulfill a criterion on parameter error, value, or value/error above or below a cutoff value.

\section{Input}

$<$ parfile $\rangle_{\text {_}}<$ parameter $>$ _comp\#.fits, any FITS file created by hfs_cube or hfs_nh3_cube, for each velocity component and parameter.

\section{Output}

$<$ parfile>_<parameter>_comp\#_blank.fits, output 2-axes FITS file, with the values of the parameter for nonflagged pixels, and NaN for flagged-out pixels.

\section{7. hfs_synt}

Auxiliary procedure to create a synthetic spectrum, with the option of adding Gaussian noise. You can select the transition, number of channels, spectral resolution, line parameters, and noise level.

\section{Output}

- hfs_synt.synt, ASCII file with the synthetic spectrum generated. The file has a header (lines beginning with "?") with the values of the parameters used for generating the synthetic spectrum.

- hfs_synt.eps, plot of the synthetic spectrum.

The author thanks Pau Estalella for suggesting the use of pseudo-random sequences, Ferran Sala for helpful discussions on the projections of a quadric, and Salvador Curiel for helping with the implementation of the multiprocessor procedures. Thanks also to Álvaro Sánchez-Monge for reading the manuscript and, together with Aina Palau, Gemma Busquet, and Carmen Juárez, for testing HfS, finding bugs, and suggesting improvements. This work has been partially supported by the Spanish MINECO grant AYA2014-57369C3 (cofunded with FEDER funds) and MDM-2014-0369 of ICCUB (Unidad de Excelencia "María de Maeztu"). 


\section{Appendix A \\ HfS Requisites and Installation}

The HfS procedures run on a Linux or Mac OS X system with a Fortran 90 compiler (for instance gfortran) and use the PGplot Graphics Subroutine Library compiled with gfortran (see Appendix B) for the graphic output and Open MPI for the multiprocessor procedures hfs_cube_mp and hfs_nh3_cube_mp.

$H f S$ can be freely downloaded as a file hfs.tgz from the Astrophysics Source Code Library, record ascl:1607.011. ${ }^{6}$ Once you have downloaded hfs.tgz, untar the file in your installation directory (as root or using sudo), for instance/usr/local/hfs,

\$mkdir/usr/local/hfs

\$mv hfs.tgz/usr/local/hfs

$\$ \mathrm{~cd} /$ usr/local/hfs

\$tar -xzvf hfs.tgz

If necessary, edit the first lines of the shell script hfs_compile to change the lines:

compiler="gfortran"

libraries="-lpgplot -1X11"

You may need to give the location of the PGplot library with the option

libraries="-lpgplot -lX11-L/usr/local/lib/pgplot"

Mac OS X users may also need to indicate the location of the X11 library:

libraries="-lpgplot-lX11-L/usr/local/lib/pgplot-L/usr/X11/lib"

Run the compile script

\$./hfs_compile

The shell script hfs_links symbolic links in \$exe_dir pointing at the HfS procedures. If necessary, change the line

exe_dir="/usr/local/bin"

and run the links script

$\$ . /$ hfs_links

Define the environment variable HFS_DIR, pointing at your installation directory. It is used by $H f S$ to find the files hfs_transitions.dat and hfs_fit.help. This can be done by adding to your .bashrc or .bash_profile the lines

\# hfs

export HFS_DIR=/usr/local/hfs

\section{Appendix B}

Installation of PGplot with Gfortran

Once you have downloaded the PGplot distribution file, follow the normal installation procedure (as root or using sudo):

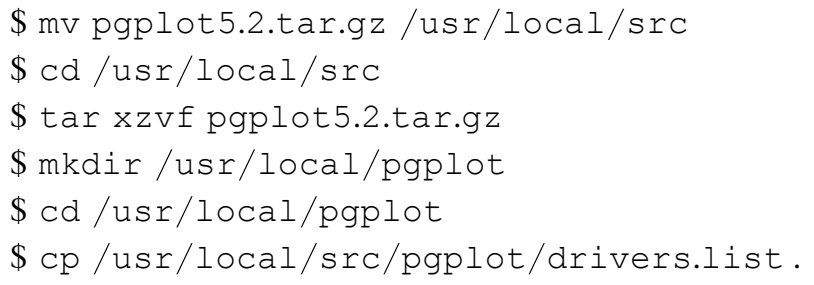


Publications of the Astronomical Society of the Pacific, 129:025003 (23pp), 2017 February

Edit the file drivers. list and uncomment (select) the drivers

/NULL (null device),

/PS, /VPS, /CPS, /VCPS (PostScript drivers), and

/XWINDOW, /XSERVE (X window drivers).

Run makemake to prepare the makefile for a Linux system with g77_gcc compiler,

\$/usr/local/src/pgplot/makemake/usr/local/src/pgplot linux g77_gcc

Edit the file makefile and replace lines 25 and 26:

$\mathrm{FCOMPL}=\mathrm{g} 77$

FFLAGC $=-\mathrm{u}-$ Wall $-\mathrm{fPIC}-\mathrm{O}$

by the following lines:

FCOMPL=gfortran

FFLAGC=-ffixed - form-ffixed - line - length - none -u -Wall -fPIC -O

Continue the normal installation procedure,

$\$$ make

\$ make clean

and, assuming that LD_LIBRARY_PATH points at/usr/local/lib,

\$cd/usr/local/lib

\$ln-s/usr/local/pgplot/libpgplot.so.

Define the environment variable PGPLOT_DIR, pointing at your installation directory. This can be done by adding to your . bashrc or .bash_profile file the lines

\# PGplot

export PGPLOT_DIR='/usr/local/pgplot'

Additionally, you can customize PGplot by adding the following lines to your .bashrc or .bash_profile:

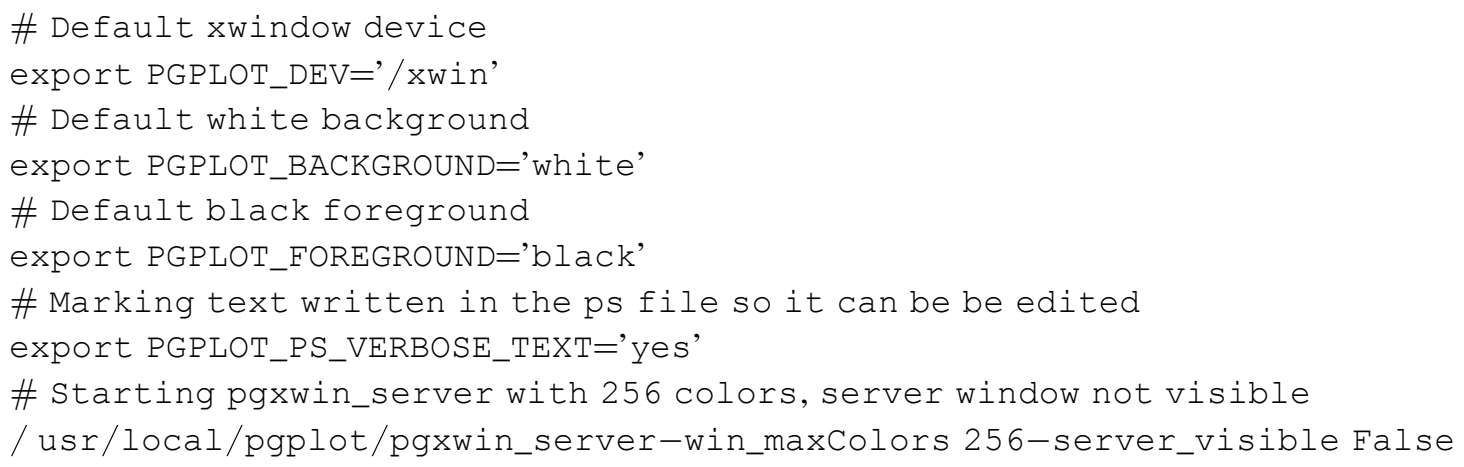




\section{Appendix C \\ Running the Multiprocessor Procedures hfs_cube_mp and hfs_nh3_cube_mp}

You need to have Open MPI installed to be able to run hfs_cube_mp or hfs_nh3_cube_mp. To run the procedures, type

$\$$ mpirun $-\mathrm{np}<\mathrm{N}>$ hfs_cube_mp $<$ parfile $>$.par

\$mpirun -np <N > hfs_nh3_cube_mp < parfile>.par

where $<\mathrm{N}>$ is the number of processors to use. To know the number (and characteristics) of processors in a Linux system, you can type

\$ cat/proc/cpuinfo

For running in more than one host, for example, in localhost and another host <otherhost>, type

$\$$ mpirun $-\mathrm{np}<\mathrm{N}>$-host localhost, <otherhost> hfs_cube_mp <parfile $>$.par

$\$$ mpirun $-\mathrm{np}<\mathrm{N}>$-host localhost, <otherhost > hfs_nh3_cube_mp <parfile $>$.par

Here, $<\mathrm{N}>$ is the total number of processors to use, distributed among the hosts listed after -host. If you want to know which are the processes run in each host, add the option -display-map.

Requisites for running in a remote host <otherhost>:

- ssh access to <otherhost>, without having to enter the password, i.e., with your local id_rsa_pub added to <otherhost>:.ssh/authorized_keys2.

- Open MPI installed in <otherhost >. Your PATH and LD_LIBRARY_PATH in <otherhost $>$ have to point at the openmpi bin and $l i b$ folders in the installation directory. For example, if openmpi is installed in /usr/local/openmpi, you can include in the file <otherhost $>$ : .bashrc the lines

export PATH=\$PATH: / usr/local/openmpi/bin

export LD_LIBRARY_PATH=\$LD_LIBRARY_PATH: / usr/local/openmpi/lib

- The same data file structure in <otherhost> and in localhost, i.e., the same directory from where you run hfs_cube_mp or hfs_nh3_cube_mp, with the same parameter file <parfile $>$.par and data files. The $\log$ files of the different processors, log/<parfile>_\#\#.log, will be written in the host where each processor runs.

\section{Appendix D}

Example of a hfs_fit Run

$\overline{\$ \text { hfs_fit nh311_thin.dat }}$

HfS_fit. HyperFine Spectra multicomponent fit

Robert Estalella, 2015/06

${ }_{\text {*******}}$ Use a terminal with at least 94 columns ${ }^{* * * * * *}$

Date: 2016/03/22 Time: 12:45:04

Data file: nh311_thin.dat

N. of data points read:

Reference channel $(\mathrm{V}=0)$ :

Channel width $\left(\mathrm{km} \mathrm{s}^{\wedge}-1\right)$ :

off-line rms:

Transition:

tau_tot/tau_m: 


\begin{tabular}{|c|c|c|c|c|}
\hline \multicolumn{5}{|c|}{$\begin{array}{ll}\text { Fit rms: } & 0.3933\end{array}$} \\
\hline \multicolumn{5}{|c|}{ Present values and search ranges $(\mathrm{ncomp}=1)$} \\
\hline Param: & Delta_V & \multicolumn{2}{|c|}{ V_lsr $A\left(1-e^{\wedge}-t \_m\right)$} & $1-e^{\wedge}-$ tau_m \\
\hline Comp: & 1 & & & \\
\hline Value: & 1.2103 & -2.9734 & 6.1716 & 0.5000 \\
\hline Range: & 1.2103 & 1.2103 & 0.3841 & 0.5000 \\
\hline
\end{tabular}

HfS fit menu

0 . Quit

1. Help

2. Read data file: nh311_thin.dat

3. Select transition: NH3 $(1,1)$

4. Hanning smoothing

5. Select plot ( 1 :data $+2:$ comp +4 :res +8 : synt $): 7$

6. Change Nksample, Final_Range: $200 \quad 0.050$

7. Change $\mathrm{Ncomp}=1$ and make initial guess

8. Change initial values or ranges

9. Fit 1 component (s) and estimate errors

Choose option $(0-9): 9$

Iteration parameters read

Nksample:

Final_Range:

Iteration para

0.050

Nseed:

Ndesc:

Nloop:

Range_Fact:

Fitting ncomp $=1$ component ( $\mathrm{s}$ )

\begin{tabular}{|c|c|c|c|c|c|c|}
\hline Loop Comp & Delta_V & "V_lsr A $\left(1-e^{\wedge}-t_{-} m\right)$ & 1-e $1-$ tau_m & rms & & \\
\hline 0 & 1 & 1.2103 & -2.9734 & 6.1716 & 0.5000 & 0.3933 \\
\hline 1 & 1 & 2.1267 & -3.9476 & 6.3392 & 0.9930 & 0.3933 \\
\hline 2 & 1 & 1.4883 & -2.9911 & 5.8081 & 0.3219 & 0.3784 \\
\hline 3 & 1 & 1.1926 & -3.1046 & 6.1852 & 0.2803 & 0.3766 \\
\hline 4 & 1 & 1.2946 & -2.9713 & 6.0164 & 0.1679 & 0.3762 \\
\hline 5 & 1 & 1.2304 & -3.0472 & 5.9933 & 0.1664 & 0.3762 \\
\hline 6 & 1 & 1.1915 & -2.9995 & 6.3971 & 0.2483 & 0.3762 \\
\hline 7 & 1 & 1.2597 & -3.0325 & 5.9275 & 0.2578 & 0.3762 \\
\hline 8 & 1 & 1.1783 & -3.0519 & 6.2819 & 0.3125 & 0.3762 \\
\hline 9 & 1 & 1.1862 & -3.0152 & 6.2391 & 0.3103 & 0.3761 \\
\hline 10 & 1 & 1.2051 & -3.0217 & 6.3489 & 0.1883 & 0.3761 \\
\hline 11 & 1 & 1.2628 & -3.0253 & 6.1733 & 0.1857 & 0.3760 \\
\hline 12 & 1 & 1.2043 & -3.0294 & 6.2455 & 0.2270 & 0.3760 \\
\hline 13 & 1 & 1.2196 & -3.0238 & 6.1633 & 0.2602 & 0.3760 \\
\hline 14 & 1 & 1.2284 & -3.0299 & 6.1945 & 0.2700 & 0.3760 \\
\hline
\end{tabular}

Error estimation in progress

\begin{tabular}{|c|}
\hline $\begin{array}{l}\text { Fit rms: } \\
\text { N. fitted par: } \\
\text { Target rms: }\end{array}$ \\
\hline Single parameters........ \\
\hline Pairs of parameters........ \\
\hline
\end{tabular}




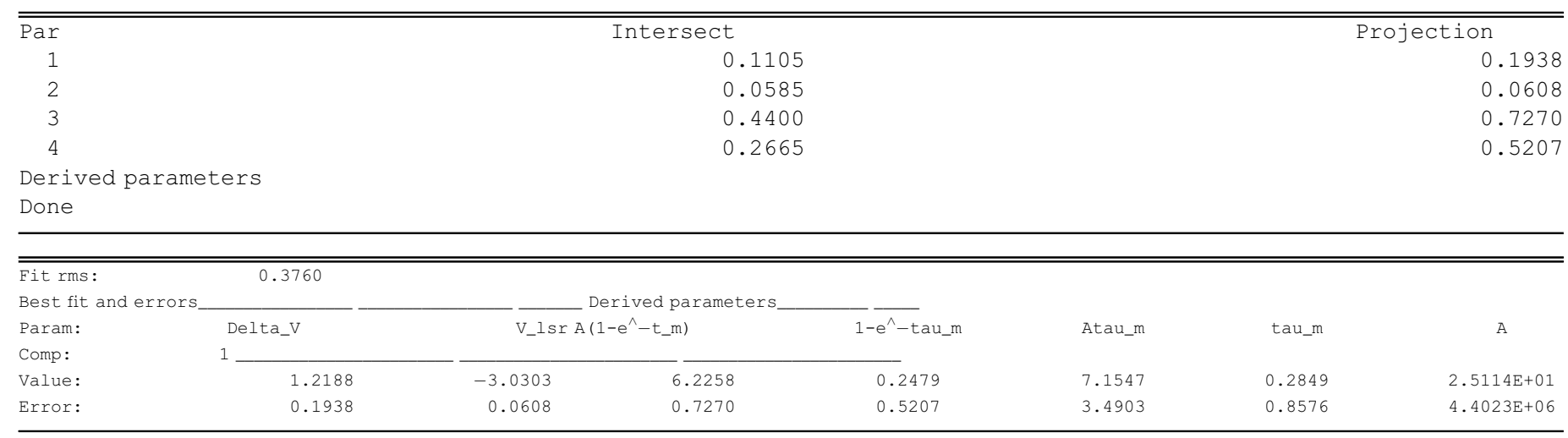

\section{Appendix E}

Example of a hfs_nh3 run

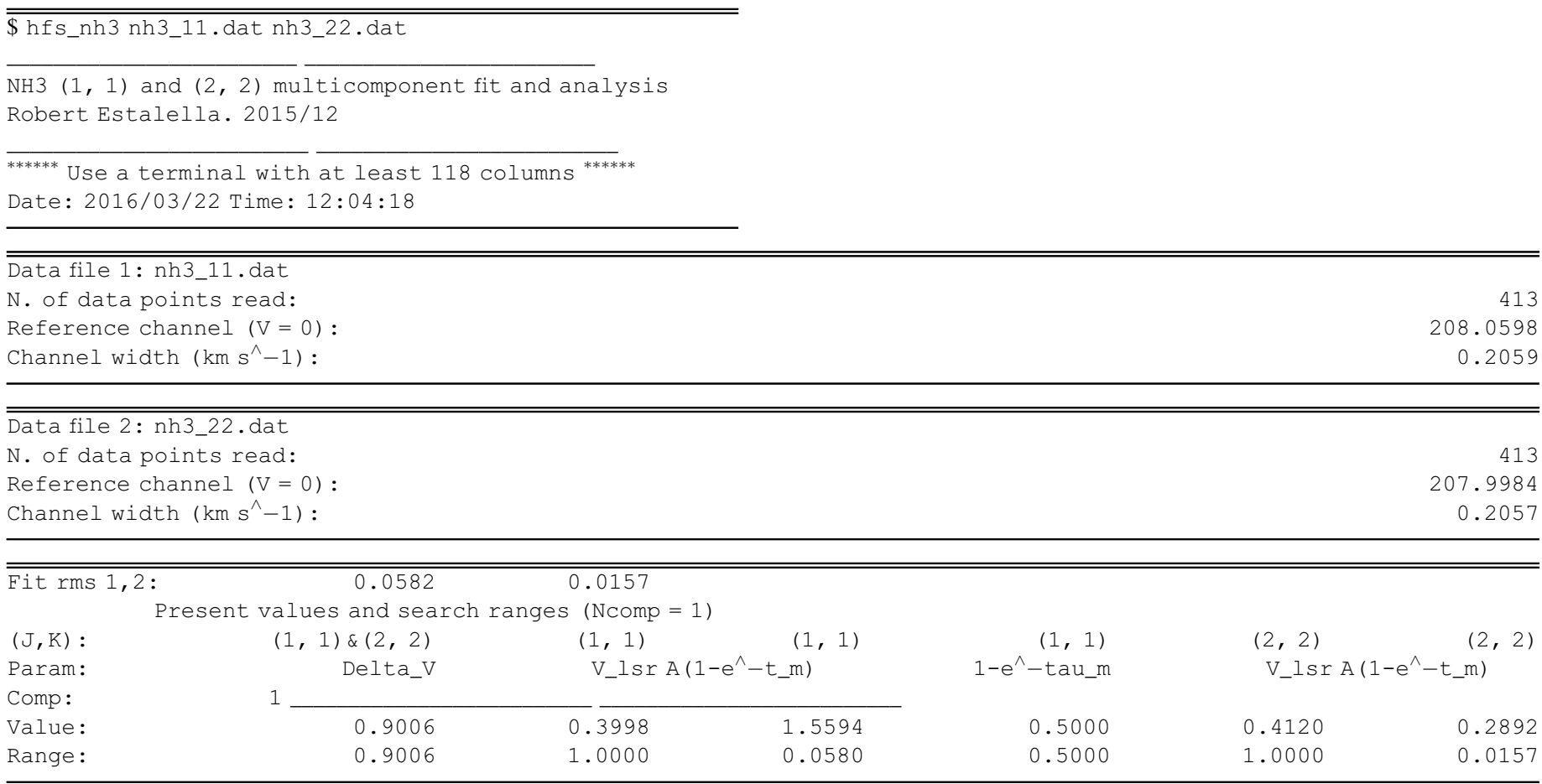

HfS NH3 $(1,1) \&(2,2)$ fit menu

0. Quit

1. Help

2. Read data files: nh3_11.dat, nh3_22.dat

3. Physical parameter estimation

4. Hanning smoothing

5. Select plot $(1:$ data $+2:$ comp $+4:$ res $+8:$ synt $): 15$

6. Change Nksample, Final_Range: $200 \quad 0.050$

7. Change $\mathrm{Ncomp}=1$ and make initial guess

8. Change initial values or ranges

9. Fit 1 component ( $S$ ) and estimate errors

Choose option (0-9): 9 
Iteration parameters read

Nksample:

Final_Range:

0.050

Iteration parameters used

Nseed:

118

Ndesc:

Nloop:

Range_Fact:

Fitting ncomp $=1$ component (s)

\begin{tabular}{|c|c|c|c|c|c|c|c|c|}
\hline \multirow{2}{*}{$\begin{array}{r}\text { Loop Comp } \\
0\end{array}$} & \multirow{2}{*}{$\frac{\text { Delta_V }}{1}$} & \multicolumn{2}{|c|}{$V_{-} I \operatorname{sr} 1 \mathrm{~A}\left(1-\mathrm{e}^{\wedge}-\mathrm{t}_{-} \mathrm{m}\right)$} & \multirow{2}{*}{$\begin{array}{c}1-e^{\wedge}-t a u \_m \\
1.5594\end{array}$} & \multicolumn{2}{|c|}{$V_{-} 1 s r 2 A\left(1-e^{\wedge}-t 2 m\right)$} & \multicolumn{2}{|l|}{ rms_tot } \\
\hline & & 0.9006 & 0.3998 & & 0.5000 & 0.4120 & 0.2892 & 0.0603 \\
\hline 1 & 1 & 1.0775 & -0.4231 & 1.5179 & 0.9924 & 1.3918 & 0.3027 & 0.0543 \\
\hline 2 & 1 & 0.8312 & 0.4102 & 1.5944 & 0.6611 & 1.3326 & 0.2791 & 0.0351 \\
\hline 3 & 1 & 0.8878 & 0.3940 & 1.6405 & 0.8201 & 0.5550 & 0.2785 & 0.0310 \\
\hline 4 & 1 & 0.7420 & 0.4763 & 1.6033 & 0.8780 & 0.0978 & 0.3075 & 0.0310 \\
\hline 5 & 1 & 0.5446 & 0.3985 & 1.5672 & 0.9798 & 1.9346 & 0.3106 & 0.0305 \\
\hline 6 & 1 & 0.6609 & 0.4237 & 1.5769 & 0.9845 & 0.3772 & 0.2785 & 0.0305 \\
\hline 7 & 1 & 0.5775 & 0.3836 & 1.5560 & 0.9914 & 0.5027 & 0.2989 & 0.0294 \\
\hline 8 & 1 & 0.5718 & 0.4494 & 1.7124 & 0.9730 & 0.2922 & 0.2766 & 0.0287 \\
\hline 9 & 1 & 0.6237 & 0.4140 & 1.5783 & 0.9651 & 0.4681 & 0.3009 & 0.0285 \\
\hline 10 & 1 & 0.5559 & 0.4449 & 1.4869 & 0.9923 & 0.2012 & 0.3003 & 0.0285 \\
\hline 11 & 1 & 0.5236 & 0.4082 & 1.5732 & 0.9881 & 0.4519 & 0.2765 & 0.0285 \\
\hline 12 & 1 & 0.5844 & 0.4423 & 1.5001 & 0.9866 & 0.4943 & 0.3013 & 0.0283 \\
\hline 13 & 1 & 0.5683 & 0.4253 & 1.5108 & 0.9860 & 0.2885 & 0.2976 & 0.0282 \\
\hline 14 & 1 & 0.5828 & 0.4155 & 1.4899 & 0.9862 & 0.4128 & 0.3338 & 0.0282 \\
\hline
\end{tabular}

Error estimation in progress

Fit rms:



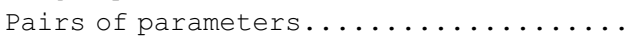

\begin{tabular}{rrr}
\hline \multicolumn{1}{l|}{} & \multicolumn{2}{c}{} \\
\hline \hline Par & Intersect & 0.0361 \\
1 & 0.0144 & 0.0099 \\
2 & 0.0094 & 0.0000 \\
3 & 0.0318 & 0.0000 \\
4 & 0.0017 & 0.0754 \\
5 & 0.0754 & 0.0725 \\
6 & 0.0696 & 07 \\
\hline
\end{tabular}

Derived parameters

Done

\begin{tabular}{|c|c|c|c|c|c|c|}
\hline Fit rms 1,2: & 0.0232 & 0.0164 & & & & \\
\hline \multicolumn{7}{|c|}{ Best fit and errors } \\
\hline$(\mathrm{J}, \mathrm{K}):$ & $(1,1) \&(2,2)$ & $(1,1)$ & $(1,1)$ & $(1,1)$ & $(2,2)$ & $(2,2)$ \\
\hline Param: & Delta_V & \multicolumn{2}{|c|}{ V_lsr $A\left(1-e^{\wedge}-t \_m\right)$} & $1-e^{\wedge}-t a u \_m$ & \multicolumn{2}{|c|}{ V_lsr $A\left(1-e^{\wedge}-t \_m\right)$} \\
\hline \multicolumn{7}{|l|}{ Comp: } \\
\hline Value: & 0.5560 & 0.4218 & 1.4801 & 0.9910 & 0.4158 & 0.3228 \\
\hline Error: & 0.0361 & 0.0099 & 0.0318 & 0.0017 & 0.0754 & 0.0725 \\
\hline \multicolumn{7}{|c|}{$\overline{\text { Derived parameters_ }}$} \\
\hline$(\mathrm{J}, \mathrm{K}):$ & $(1,1)$ & $(1,1)$ & $(2,2)$ & $(2,2)$ & & $(1,1) \&(2,2)$ \\
\hline Param: & Atau_m & tau_m & Atau_m & tau_m & & A \\
\hline \multicolumn{7}{|l|}{ Comp: } \\
\hline Value: & 7.0371 & 4.7118 & 0.3638 & 0.2436 & & $1.4935 \mathrm{E}+00$ \\
\hline Error: & 0.3068 & 0.1865 & 0.0927 & 0.0623 & & $3.2230 \mathrm{E}-02$ \\
\hline
\end{tabular}




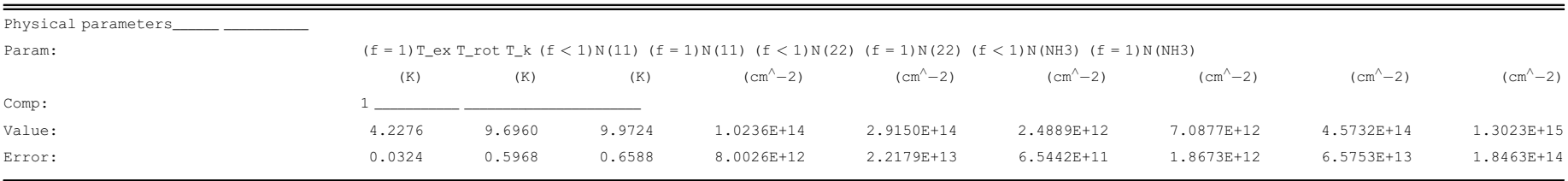

Appendix F

Examples of $\boldsymbol{H} \boldsymbol{f S}$ input files

F.1. <ile_list>.par

\begin{tabular}{|c|c|}
\hline "NH3 $(1,1) "$ & ! Transition \\
\hline 4000.05 & ! Nksample, Final_Range \\
\hline 00117+6412.nh311. clump1.spt & ! 1st data file \\
\hline AFGL5142.nh311. clump1. dat & ! 2nd data file \\
\hline CepA.nh311.clump1.spt & $! \ldots$ \\
\hline ON1.nh311. clump1.spt & ! Last data file \\
\hline
\end{tabular}

\section{F.2. Parameter File for hfs_cube_sp and hfs_cube_mp}

\begin{tabular}{|c|c|}
\hline "N2H+(1-0)" & ! Transition \\
\hline "n2hp-merged-lmv-clean.fits" & ! Input data cube file \\
\hline 0.068 .0 & ! rms, minimum SNR to analyze the spectrum \\
\hline 1 & ! Number of components \\
\hline 104108 & ! Range of channels to search for peak \\
\hline 0 & ! Hanning filter half-width (chan) : $0=$ no; $>0=$ yes \\
\hline 0 & ! Boxcar smoothing radius (pixels) : $0=$ no; $>0=$ yes \\
\hline 202364 & ! Xpix_ini, xpix_fin, Xpix_inc \\
\hline 202364 & ! Ypix_ini, Ypix_fin, Ypix_inc \\
\hline 4000.05 & ! Nksample, Final_Range \\
\hline
\end{tabular}

\section{F.3. Parameter File for hfs_nh3_cube_sp and hfs_nh3_cube_mp}

\begin{tabular}{|c|c|}
\hline "11287_11_Kv.fits" & ! $(1,1)$ input NH3 $(1,1)$ data cube file \\
\hline 0.304 .0 & ! $(1,1)$ rms, minimum SNR to analyse the spectrum \\
\hline 3 & ! Number of components \\
\hline 272930323335 & ! $(1,1)$ range of channels to search for peak \\
\hline "11287_22_Kv.fits" & ! $(2,2)$ input $\mathrm{NH} 3(2,2)$ data cube file \\
\hline 0.304 .0 & ! $(2,2)$ rms, minimum SNR to analyse the spectrum \\
\hline 0 & ! Hanning filter half-width (chan) : $0=$ no; $>0=$ yes \\
\hline 1 & ! Boxcar smoothing radius (pixels) : $0=$ no; $>0=$ yes \\
\hline 1283843 & $!(1,1) \&(2,2)$ Xpix_ini, Xpix_fin, Xpix_inc \\
\hline 1283843 & $!(1,1) \&(2,2)$ Ypix_ini, Ypix_fin, Ypix_inc \\
\hline 4000.05 & ! Nksample, Final_Range \\
\hline
\end{tabular}


Publications of the Astronomical Society of the Pacific, 129:025003 (23pp), 2017 February

Appendix G

Examples of $\boldsymbol{H} f \mathrm{~S}$ Output Files

G.1. Header and First Lines of <source> synt

\begin{tabular}{|c|c|c|c|c|}
\hline \multicolumn{5}{|c|}{ ! Created by HfS_nh3 } \\
\hline \multicolumn{2}{|l|}{ ! DATE } & $=$ & & $2016 / 01 / 12$ \\
\hline \multicolumn{2}{|l|}{ !TIME } & $=$ & & $12: 59: 00$ \\
\hline \multicolumn{2}{|l|}{ ! TRANSITION } & $=$ & & NH3 $(2,2)$ \\
\hline \multicolumn{2}{|l|}{ ! NCHAN } & $=$ & & 120 \\
\hline \multicolumn{2}{|l|}{ ! DVCHAN } & $=$ & & 0.30851 \\
\hline \multicolumn{2}{|l|}{ ! NCOMP } & $=$ & & 2 \\
\hline \multicolumn{2}{|l|}{ ! DVLINE_1 } & $=$ & & 1.05310 \\
\hline \multicolumn{2}{|l|}{ !VLSR__ 1} & $=$ & & -18.38200 \\
\hline \multicolumn{2}{|l|}{ ! A TAU_M_1 } & $=$ & & 0.00226 \\
\hline \multicolumn{2}{|l|}{ ! TAU_M__1 } & $=$ & & 0.13445 \\
\hline \multicolumn{2}{|l|}{ ! DVLINE_2 } & $=$ & & 0.00000 \\
\hline \multicolumn{2}{|l|}{ !VLSR__ 2} & $=$ & & 0.00000 \\
\hline \multicolumn{2}{|l|}{ ! A*TAU_M_2 } & $=$ & & 0.00000 \\
\hline \multicolumn{2}{|l|}{ ! TAU_M_L_ 2} & $=$ & & 0.00000 \\
\hline \multirow[t]{9}{*}{$\overline{!}$} & "VELOCITY & S SYNTHETIC & "COMP_1 & 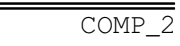 \\
\hline & -36.50216 & 0.00002 & 0.00002 & 0.00000 \\
\hline & -36.19365 & 0.00006 & 0.00006 & 0.00000 \\
\hline & -35.88514 & 0.00011 & 0.00011 & 0.00000 \\
\hline & -35.57662 & 0.00014 & 0.00014 & 0.00000 \\
\hline & -35.26811 & 0.00011 & 0.00011 & 0.00000 \\
\hline & -34.95960 & 0.00006 & 0.00006 & 0.00000 \\
\hline & -34.65109 & 0.00002 & 0.00002 & 0.00000 \\
\hline & -34.34258 & 0.00000 & 0.00000 & 0.00000 \\
\hline
\end{tabular}

G.2. Header and First Lines of <source $>_{-}<$xoffset $>$_ $<$yoffset $>$.spec

! Created by HfS_view






\section{G.3. <parfile>_comp\#.out}

! Created by HfS_cube

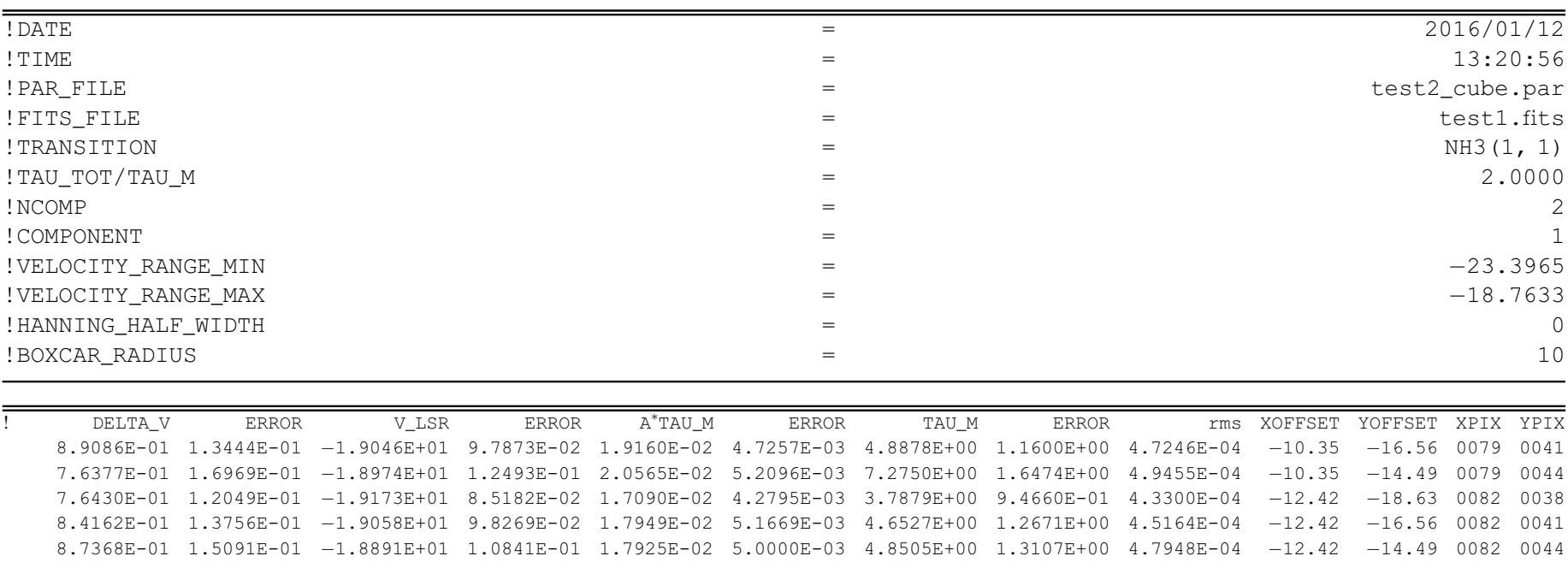

\section{References}

Anglada, G., Estalella, R., Mauersberger, R., et al. 1995, ApJ, 443, 682

Avni, Y. 1976, ApJ, 210, 642

Cantó, J., Curiel, S., \& Martínez-Gómez, E. 2009, A\&A, 501, 1259

Danby, G., Flower, D. R., Valiron, P., Schilke, P., \& Walmsley, C. M. 1988, MNRAS, 235, 229

Estalella, R. 2016, HfS: Hyperfine Structure Fitting Tool, Astrophysics Source Code Library, ascl:1607.011

Estalella, R., \& Anglada, G. 1997, Introducción a la Física del Medio Interestelar, Col-lecció Textos Docents, n. 50 (2nd Ed.; Spain: Edicions de la Universitat de Barcelona)

Estalella, R., López, R., Anglada, G., et al. 2012, AJ, 144, 61

Faure, A., Hily-Blant, P., Le Gal, R., Rist, C., \& Pineau des Forêts, G. 2013 , ApJ, 770, L2

Goddi, C., Zhang, Q., \& Moscadelli, L. 2015, A\&A, 573, A108

Halton, J. H. 1964, Commun. ACM, 7, 701

Ho, P. T. P., \& Townes, C. H. 1983, ARAA, 21, 239
Kukolich, S. G. 1967, Phys. Rev., 156, 83

Mangum, J. G., \& Shirley, Y. L. 2015, PASP, 127, 266

Maret, S., Faure, A., Scifoni, E., \& Wiesenfeld, L. 2009, MNRAS, 399, 425

McConnell, A. J. 2011, Applications of Tensor Analysis (New York: Dover Publications)

Osorio, M., Anglada, G., Lizano, S., \& D’Alessio, P. 2009, ApJ, 694, 29

Palau, A., Estalella, R., Girart, J. M., et al. 2014, ApJ, 785, 42

Poynter, R. L., \& Kakar, R. K. 1975, ApJ, 29, 87

Rosolowsky, E. W., Pineda, J. E., Foster, J. B., et al. 2008, ApJSS, 175, 509

Sánchez-Monge, Á., Palau, A., Fontani, F., et al. 2013, MNRAS, 432, 3288

Sepúlveda, I., Anglada, G., Estalella, R., et al. 2011, A\&A, 527, A41

Svoboda, B. E., Shirley, Y. L., Battersby, C., et al. 2016, ApJ, 822, 59

Sobol, I. 1967, USSR Computational Mathematics and Mathematical Physics, 7,86

Swift, J. J., Welch, W. J., \& Di Francesco, J. 2005, ApJ, 620, 823

Walmsley, C. M., \& Ungerechts, H. 1983, A\&A, 122, 164

Wall, J. V., \& Jenkins, C. R. 2003, Practical Statistics for Astronomers (Cambridge: Cambridge Univ. Press) 